\title{
Mechanical Behavior of Buried Pipelines Subjected to Faults
}

\author{
Xiaolong Wei $\mathbb{D},{ }^{1}$ Wenshuai Jiao, ${ }^{2}$ Xi Zeng, ${ }^{3}$ Danfu Zhang, ${ }^{4}$ and Guofeng Du $\mathbb{D}^{2}$ \\ ${ }^{1}$ School of Petroleum Engineering, Yangtze University, Wuhan 430100, China \\ ${ }^{2}$ School of Urban Construction, Yangtze University, Jingzhou 434000, China \\ ${ }^{3}$ China Construction Third Bureau Second Engineering Co., Ltd., Wuhan 430000, China \\ ${ }^{4}$ China Construction Third Bureau First Engineering Co., Ltd., Wuhan 430040, China \\ Correspondence should be addressed to Guofeng Du; 201873005@yangtzeu.edu.cn
}

Received 6 March 2021; Revised 3 June 2021; Accepted 9 June 2021; Published 23 June 2021

Academic Editor: Valeria Vignali

Copyright ( $) 2021$ Xiaolong Wei et al. This is an open access article distributed under the Creative Commons Attribution License, which permits unrestricted use, distribution, and reproduction in any medium, provided the original work is properly cited.

\begin{abstract}
The length of buried pipelines usually extends thousands of meters or more in engineering, and it is difficult to carry out full-scale tests in the laboratory. Therefore, considering the seriousness of pipeline damage and the difficulty of operating tests and other test limitations, it is necessary to develop a reasonable method to simplify the length of the model for a practical lab test. In this research, an equivalent spring model was established to simulate the small deformation section of the pipeline far away from the fault and the effect of fault displacements, pipeline diameters, wall thicknesses, buried depths, soil materials, and spring constraints on the mechanical properties of pipelines was analyzed. Based on the finite element model using ABAQUS software, the results of the shell model with fixed boundary at both ends were compared; in addition, the dynamic effect of pipelines was investigated. The results show that the two-end spring device can better control the size of the test model and enhance the reliability of the test results. The vibration response of the pipeline mainly depends on the inconsistent movement of soil at both ends of the fault. The analysis results show that choosing a larger pipeline diameter, smaller buried depth, noncohesive backfill soil, and spring with a smaller elastic coefficient is beneficial to reduce pipeline strain and resist pipeline deformation. A simplified formula of the axial compressive strain of buried pipelines across oblique-slip fault is obtained.
\end{abstract}

\section{Introduction}

The San Fernando earthquake in 1971 gave birth to the lifeline earthquake project $[1,2]$, and then much research has been conducted to understand further the action mechanism of the pipeline under fault movement. Some experimental studies were carried out, mainly including the small-scale test based on shaking tables or the centrifuge device and the full-scale test. Erami et al. [3] carried out a full-scale test to study the pipe-soil interaction equation. The experimental results were compared with the results of the computeraided analysis. The results show that the nature of the soil and the connection nodes have a great influence on the pipesoil interaction. Feng et al. [4] conducted static and dynamic seismic tests on buried pipelines across faults. The results showed that there was no significant difference between the responses of pipelines under the displacement of dynamic test and those under the static test. Demirci et al. [5] conducted a series of experiments to analyze the behavior of continuous buried pipelines subjected to reverse fault motion and developed a novel experimental device for studying pipelines crossing reverse faults. Zhang et al. [6] carried out a full-scale test to study the effect of soil settlement on the pipeline. Zhang et al. $[7,8]$ conducted the shaking table test of buried pipelines under the strike-slip fault and found that the dynamic effect of the pipeline-soil system itself has little effect on the mechanical properties of pipelines. Zhang et al. [9] studied the force process of buried pipelines under the fault through the test and the numerical simulation. Tang et al. [10] carried out the centrifuge test to simulate the reaction characteristics of buried pipelines under the reverse fault. Li et al. [11] used the shaking table device to analyze the field seismic response rules under the consistent and nonconsistent seismic actions. Oskouei et al. [12] conducted multiple sets of tests to evaluate the impact of buried depth on the buried steel pipelines crossing the strike-slip faults 
and analyzed the effects of different soil deformation forms on the buried pipeline. The full-scale test and the small-scale test based on shaking table device were carried out, respectively, in the Cornell University and the Rensselaer Polytechnic Institute to simulate the fault movement and obtain the mechanical properties of buried pipelines. The above-mentioned studies were from 2005 to 2009 and a series of important results were obtained [13-17]. Kim et al. [18] used the full-scale test device of Cornell University to study the response behaviors of buried concrete pipelines subjected to faults and found that the main failure of pipelines occurs at the joint near the fault. Rojhani et al. $[19,20]$ used the self-made centrifuge test device connected by springs at the end of the pipeline to carry out the simulation tests of stainless steel pipelines under the normal and reverse faults action and found that the test results are in good agreement with the analytical values. Sim et al. [21] carried out the experimental studies of pipelines under the strike-slip and normal faults based on the shaking table device. Rofooei et al. $[22,23]$ carried out a full-size test to study the deformation and buckling behaviors of steel pipelines and HDPE pipelines under the reverse fault simulated by the soil-box and hydraulic driving device.

Relevant research literature [24] has shown that the static test can be used to study the mechanical performance of pipelines subjected to fault at the low frequency of ground motion. Moreover, both ends of the pipeline are usually assumed fixed ends in most experiments and few experiments were conducted to study the pipe-soil constraints far from the fault. In view of this, an equivalent spring model was established to simulate the small deformation section of the pipeline far away from the fault and 27 pipelines crossing different faults were investigated. In addition, the effects of pipeline diameter, buried depth, soil materials, and spring with different elastic coefficients on the mechanical properties of the pipeline were analyzed.

\section{Experimental Study}

2.1. Test Specimens and Soil-Box. Twenty-seven buried pipelines made of Q235 hot-rolled steel were designed, fabricated, and tested to investigate the effects of different parameters on their mechanical behavior subjected to faults. The geometric parameters of each specimen are given in Table 1. All the specimens had the same length of $3.0 \mathrm{~m}$, and both ends of each pipeline were welded with a nut, which was connected with the spring device and fixed on the soilbox, as shown in Figure 1.

A soil-box, $\quad 3.0 \mathrm{~m} \quad$ (length) $\times 1.0 \mathrm{~m} \quad$ (width) $\times 1.1 \mathrm{~m}$ (height), was designed to simulate the movement of diverse types of faults, which was removable and mainly contained four parts, that is, fixed and staggered plate, soil-retaining side panel, spring device, and portal steel frame, as shown in Figure 2. The soil-box could be loaded by vertical or horizontal jack. The soil-retaining side panel could rotate around the bottom screw to simulate different angles crossing faults. In practical engineering, the length of buried pipeline between stations usually extends thousands of meters; however, only a small length of the pipeline was taken out as a
TABLE 1: Details of specimens.

\begin{tabular}{lccccc}
\hline Test coupons & $\theta\left(^{\circ}\right)$ & $k(\mathrm{~N} / \mathrm{m})$ & $D(\mathrm{~mm})$ & $H(\mathrm{~m})$ & $t(\mathrm{~mm})$ \\
\hline NC-1 & 75 & 4729 & 32 & 0.3 & 2.0 \\
NC-2 & 75 & 4729 & 32 & 0.6 & 2.0 \\
NC-3 & 75 & 4729 & 40 & 0.3 & 2.0 \\
NC-4 & 90 & 4729 & 32 & 0.3 & 2.0 \\
NC-5 & 90 & 4729 & 32 & 0.6 & 2.0 \\
NC-6 & 90 & 4729 & 40 & 0.3 & 2.0 \\
NS-7 & 90 & 4729 & 32 & 0.3 & 2.0 \\
NS-8 & 90 & 2156 & 32 & 0.3 & 2.0 \\
RC-1 & 105 & 4729 & 32 & 0.3 & 2.0 \\
RC-2 & 105 & 4729 & 32 & 0.6 & 2.0 \\
RC-3 & 105 & 4729 & 40 & 0.3 & 2.0 \\
SC-1 & 90 & 4729 & 32 & 0.3 & 2.0 \\
SC-2 & 90 & 4729 & 32 & 0.6 & 2.0 \\
SC-3 & 90 & 4729 & 40 & 0.3 & 2.0 \\
OC-1 & 90 & 4729 & 40 & 0.3 & 1.0 \\
OC-2 & 90 & 4729 & 40 & 0.6 & 1.0 \\
OC-3 & 90 & 4729 & 40 & 0.3 & 2.0 \\
OC-4 & 90 & 4729 & 32 & 0.3 & 1.0 \\
OC-5 & 90 & 4729 & 32 & 0.6 & 1.0 \\
OC-6 & 90 & 4729 & 32 & 0.3 & 2.0 \\
OC-7 & 90 & 4729 & 25 & 0.3 & 1.0 \\
OC-8 & 90 & 4729 & 25 & 0.6 & 1.0 \\
OS-9 & 90 & 4729 & 32 & 0.6 & 1.0 \\
OS-10 & 90 & 4729 & 32 & 0.3 & 1.0 \\
OS-11 & 90 & 4729 & 32 & 0.3 & 2.0 \\
OS-12 & 90 & 4729 & 25 & 0.3 & 1.0 \\
OS-13 & 90 & 12980 & 25 & 0.3 & 1.0 \\
\hline Note & & & 0.3 \\
\hline
\end{tabular}

Note. The first letters in test coupons "N," "R," "S," and "O" stand for various fault types referring to the normal fault, the reverse fault, the strikeslip fault, and the oblique-slip fault, respectively. The second letters in test coupons " $C$ " and " $S$ " stand for various soil materials referring to the clay, and the medium sand, respectively; " $\theta$," " $k$," " $D$," " $H$," and " $t$ " stand for angles crossing the faults, elasticity coefficients at both ends of the pipelines, diameters of the pipelines, buried depths of the pipelines, and wall thicknesses of the pipelines, respectively.

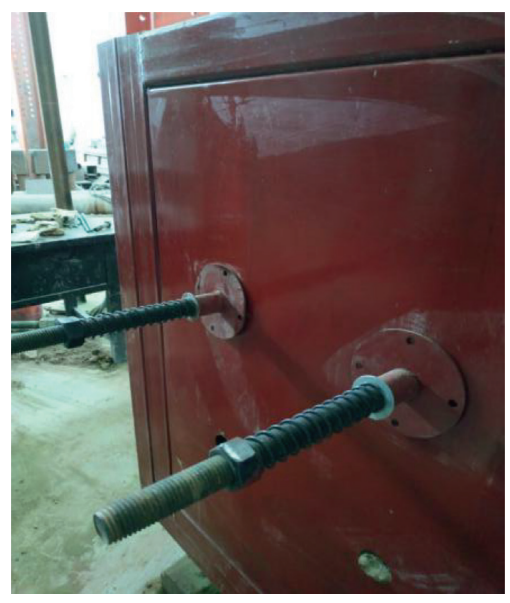

Figure 1: Spring device.

specimen when the mechanical behavior of the pipeline was tested in the laboratory; the purpose of spring device was to make the pipeline far away from the fault to be stretchable. When selecting the spring coefficient, the stress value of the pipe section at different points from the fault is obtained 


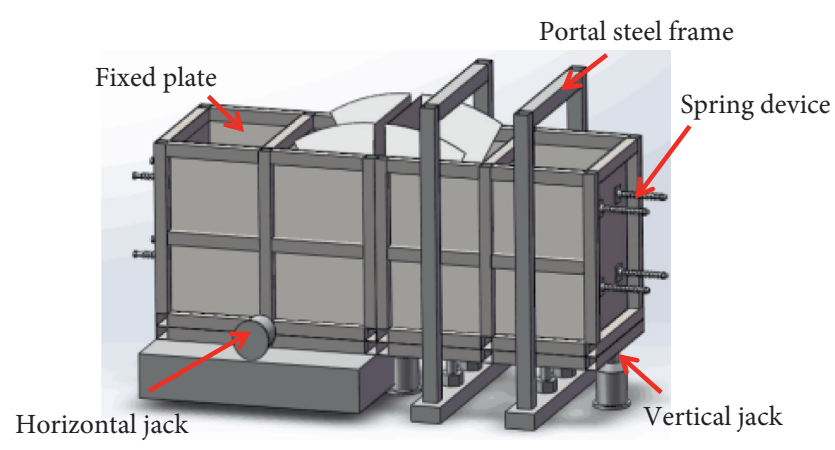

Figure 2: Soil-box test device.

through finite element simulation. The axial force at the pipe section is obtained and substituted into the expression of the equivalent spring of the small deformation section of the pipe [24] to determine the spring coefficient of elasticity. The portal steel frame was used to restrict the horizontal displacement in the process of jack-up loading.

2.2. Material Properties of Steel Pipelines and Soils. Tensile coupon tests were conducted to determine the mechanical properties of steel pipelines. The material properties of the steel tubes were determined by tensile tests at ambient temperature. The steel coupons were extracted from the pipelines in the longitudinal direction of the untested specimens. The material properties of the steel obtained from the tensile coupon tests are listed in Table 2, where $E_{0}$ is the elastic modulus, $f_{y}$ is the yield strength, $f_{u}$ is the ultimate strength, and $\mu$ is the Poisson ratio. Based on a series of unconsolidated-undrained triaxial tests, the material properties of soils adopted by clay or medium sand were measured, as shown in Table 3, where $\varphi$ is the internal friction angle, $c$ is the cohesive force, $\rho$ is the density, and $\mu$ is the Poisson ratio.

2.3. Experiment Procedures. The soil-box was loaded by horizontal or vertical jack to simulate the fault movement in this experiment; the load based on the displacement control method ranged from $0 \mathrm{~mm}$ to $140 \mathrm{~mm}$, which was divided into 14 steps, and each loading displacement was $10 \mathrm{~mm}$. When simulating normal or reverse fault, the fixed plate was fixed on the base, and two vertical jacks at the bottom of the staggered plate were used to produce vertical displacement. When simulating strike-slip fault, a horizontal jack in the middle of the staggered plate (at this point, the fixed plate was regarded as a staggered plate) was used to produce horizontal displacement. When simulating oblique-slip fault, horizontal and vertical jacks were used to load in parallel to simulate the movement along the angle of $45^{\circ}$ with fault trend and tendency. Figure 3 presents four types of fault movement.

Since the large deformation section of the pipeline was mainly investigated in this article, strain gauges near the fault area were densely arranged. As shown in Figure 4, 18 strain gauges are symmetrically bonded along the outer surface of the pipeline in the axial direction to measure the
TABLE 2: Material properties of steel pipelines.

\begin{tabular}{lcccc}
\hline Test coupons $(D \times t)$ & $f_{y}(\mathrm{MPa})$ & $f_{u}(\mathrm{MPa})$ & $E_{0}(\mathrm{GPa})$ & $\mu$ \\
\hline $25 \times 1.0$ & 278.0 & 365.2 & 203 & 0.3 \\
$32 \times 1.0$ & 279.5 & 364.9 & 203 & 0.3 \\
$32 \times 2.3$ & 292.8 & 392.3 & 204 & 0.3 \\
$40 \times 1.0$ & 282.5 & 365.4 & 202 & 0.3 \\
$40 \times 2.3$ & 290.7 & 386.1 & 203 & 0.3 \\
\hline
\end{tabular}

Table 3: Material properties of soils.

\begin{tabular}{lcccc}
\hline Soils & $\varphi\left(^{\circ}\right)$ & $c(\mathrm{kPa})$ & $\rho\left(\mathrm{g} \cdot \mathrm{cm}^{-3}\right)$ & $\mu$ \\
\hline Clay & 7.8 & 35.5 & 1.88 & 0.35 \\
Medium sand & 30.2 & 3.4 & 2.32 & 0.3 \\
\hline
\end{tabular}

strain distribution. Due to the small diameter of the pipe selected in the test, the accurate attach positions were marked and the surfaces of these positions were cleaned with alcohol before the strain gauges were mounted with epoxy resin. Each of the attached gauges was covered with a circle of tape for protection and tested with an ohmmeter every time before the experiment. The strain data of pipelines were collected by DH3816 static strain acquisition system, which can satisfy 60 measuring points at the same time. In addition, the deformation data of pipelines were indirectly measured by the vertical deformation of the rigid rod that was bonded with an adhesive and extended out of the soil surface; the experimental measurement site is presented in Figure 5.

\section{Experimental Results and Discussion}

3.1. Effect of the Fault Displacements. Figures 6(a) and 6(b) show the soil and pipelines deformation under the fault, respectively. The relationship of the strain and the fault displacement is presented in Figures 7-11. It can be seen that the obtained strain curves of specimens NC-1, NC-4, RC-1, SC-1, and OC- 6 show similar changes in the rule. The strain values at different measuring points increase with the increase of fault displacement, and the point of the maximum strain is near both sides of the fault rather than being in the fault area; for example, the tensile strain at point 8-1 and the compressive strain at point 2-1 in Figure 7(a) are maximum as the fault displacement reaches in $140 \mathrm{~mm}$, which can be observed in a similar situation in other specimens, thus showing that pipelines in certain areas near faults are most vulnerable to damage.

Compared with the above strain curve change, there are some differences in points. For example, the measurement point 5-1 in Figure 7 is the pipe section at the fault. When the fault displacement is less than $60 \mathrm{~mm}$, the upper side of the pipeline will generate axial compressive stress under the action of shear force, and within this range, the axial compressive stress will increase with the fault displacement. At the same time, because the pipe section at measuring point $5-1$ is restrained by the enhanced dislocation disk and fixed disk, the upper side compressive strain of the pipeline decreases with the further increase of the fault displacement, and the pipeline begins to show axial tensile strain due to the reverse tensile stress. The same principle can explain the 


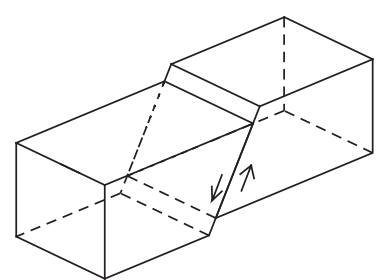

(a)

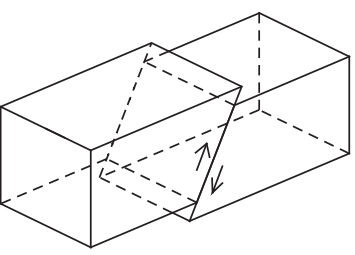

(b)

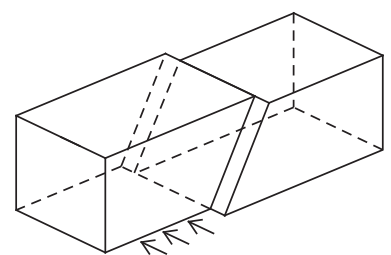

(c)

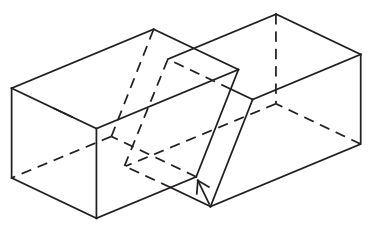

(d)

FIGURE 3: Different types of faults: (a) normal fault; (b) reverse fault; (c) strike-slip fault; (d) oblique-slip fault.

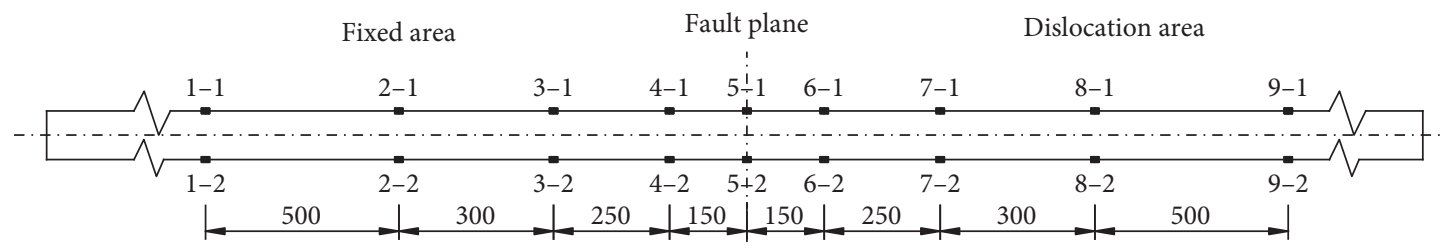

FIGURE 4: Layout of strain gauge.

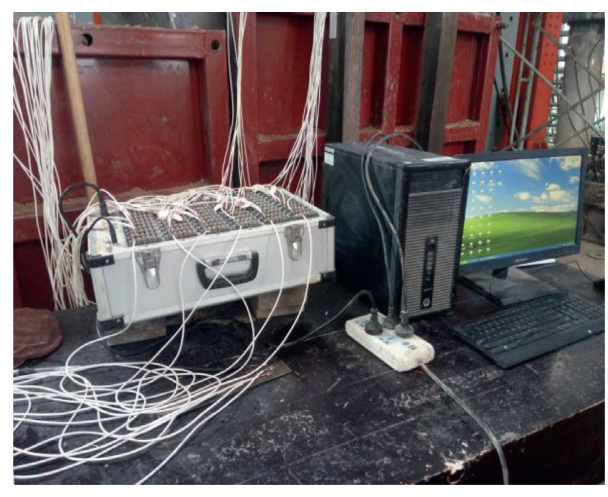

(a)

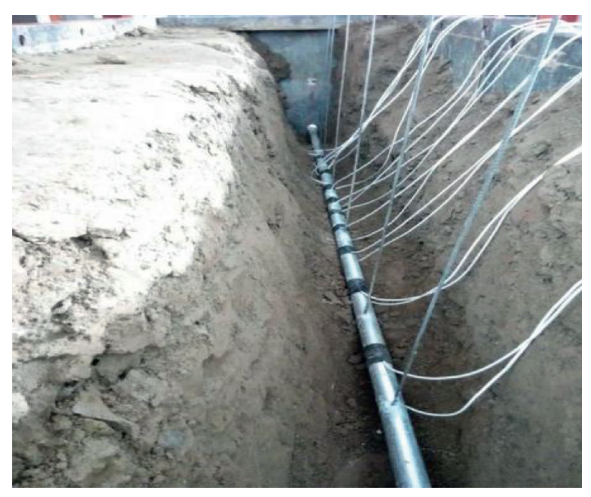

(b)

Figure 5: Test measurement: (a) strain measurement; (b) deformation measurement.

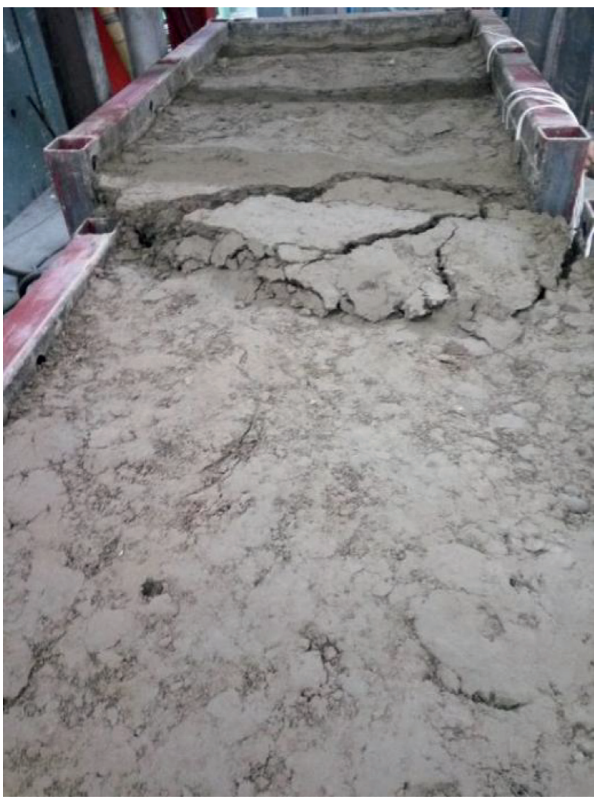

(a)

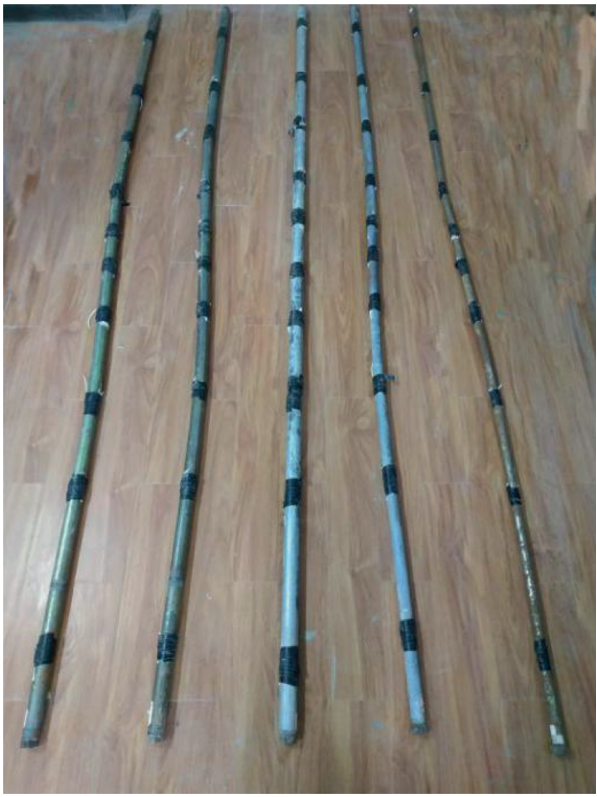

(b)

FIgURE 6: Deformation of soil and pipelines: (a) soil deformation; (b) pipelines deformation. 

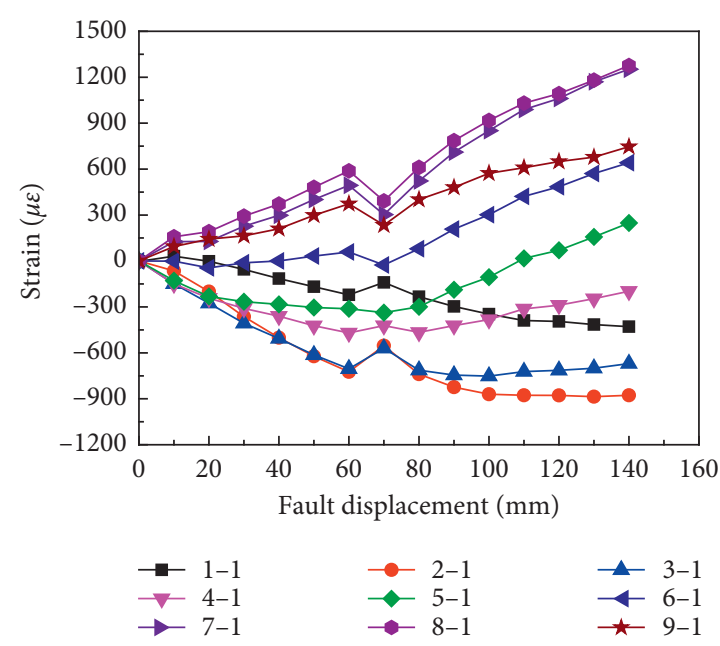

(a)

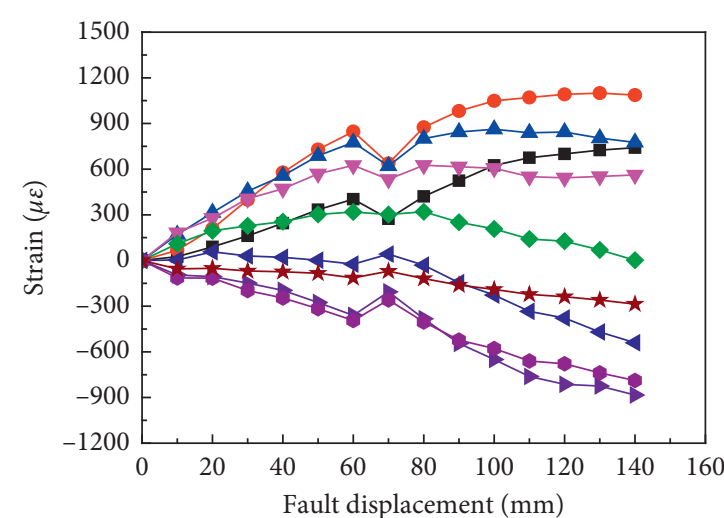

$\rightarrow-1-2$
$\rightarrow-4-2$
$\rightarrow-7-2$

$\longrightarrow 2-2$
$\longrightarrow-5-2$
$\rightarrow-8-2$

$-3-2$

$\leftarrow 6-2$

(b)

FIGURE 7: Strain-fault displacement curves of NC-1: (a) upper side; (b) underside.

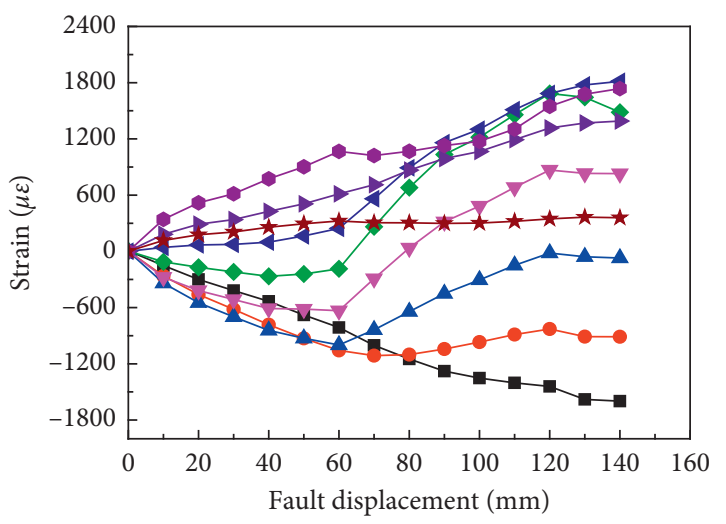

$\begin{array}{lll}\rightarrow-1-1 & \rightarrow 2-1 & \rightarrow 3-1 \\ \rightarrow-4-1 & \rightarrow 5-1 & \because-6-1 \\ \rightarrow-7-1 & \rightarrow-1 & \text { ॠ } 9-1\end{array}$

(a)

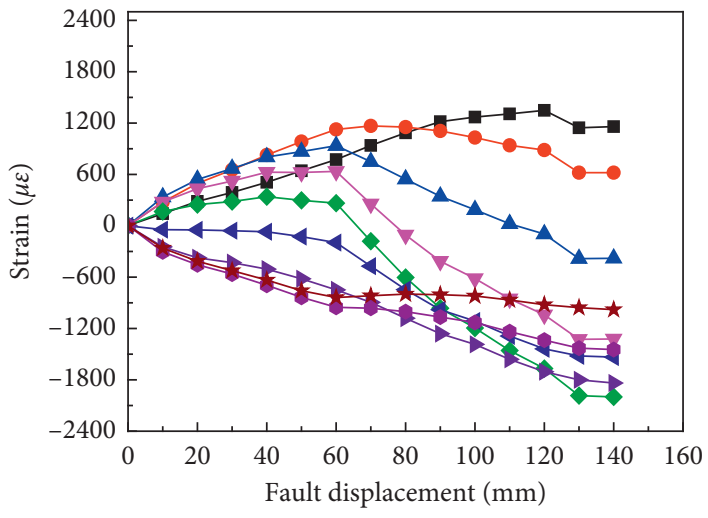

$\rightarrow-1-2$

$\longrightarrow$
$\rightarrow-7-2$
$\rightarrow-7-2$

$\rightarrow 2-2$

$\longrightarrow$ 5-2

$-3-2$

(b)

FIgURE 8: Strain-fault displacement curves of NC-4: (a) upper side; (b) underside.
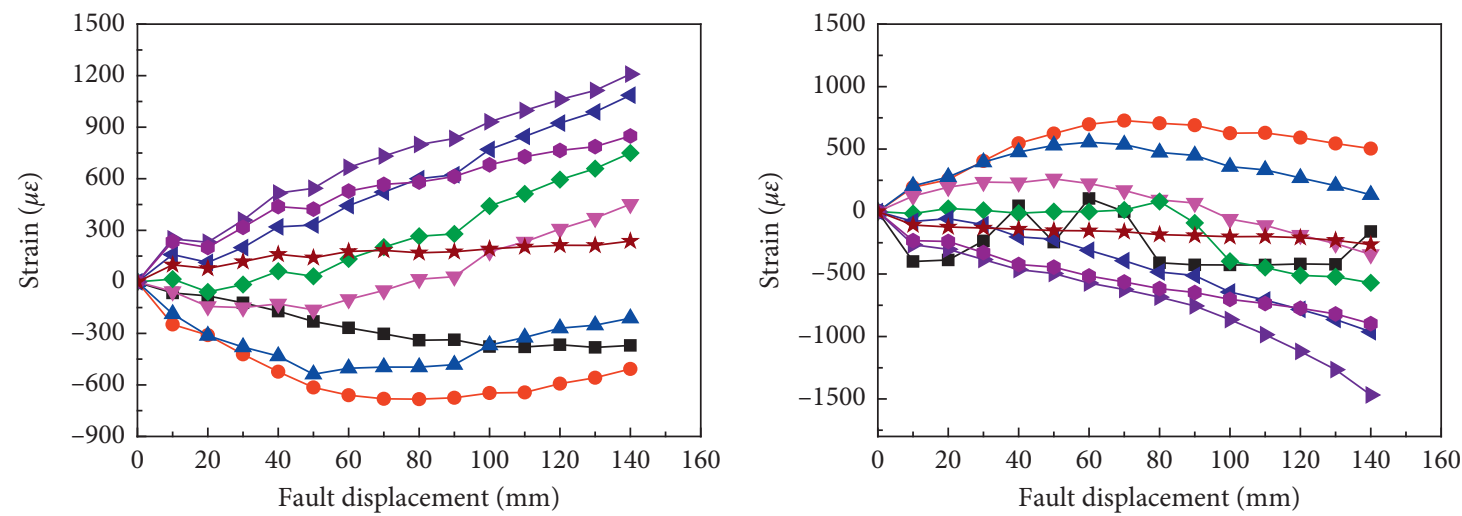

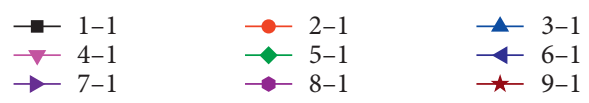

(a)

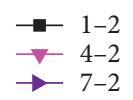

$\longrightarrow$
$\rightarrow-7-2$ $\longrightarrow$
$-\quad 2-2$
$-5-2$
$-8-2$

(b)

Figure 9: Strain-fault displacement curves of RC-1: (a) upper side; (b) underside. 


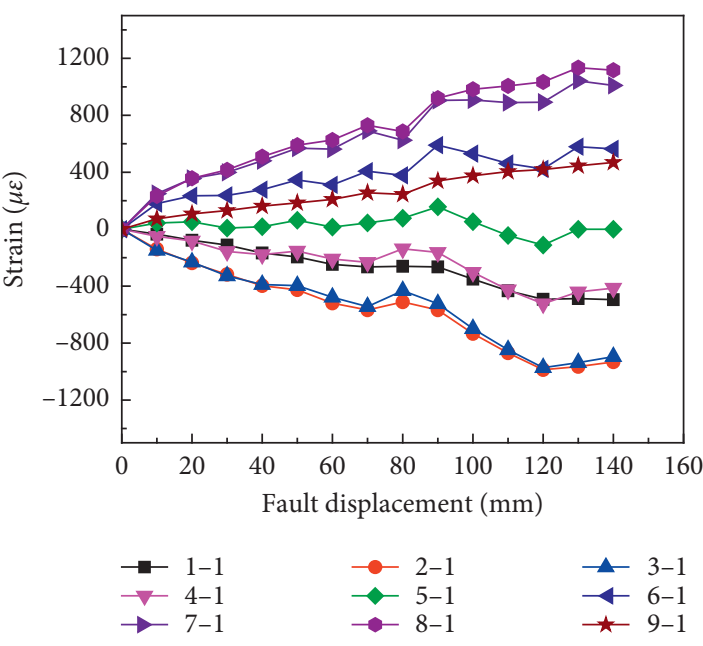

(a)
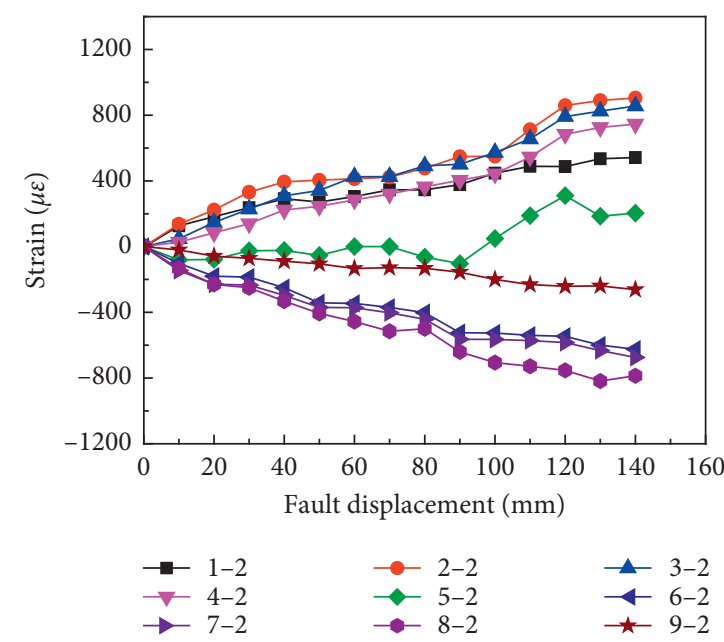

$-3-2$

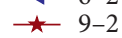

(b)

FIGURE 10: Strain-fault displacement curves of SC-1: (a) left side; (b) right side.

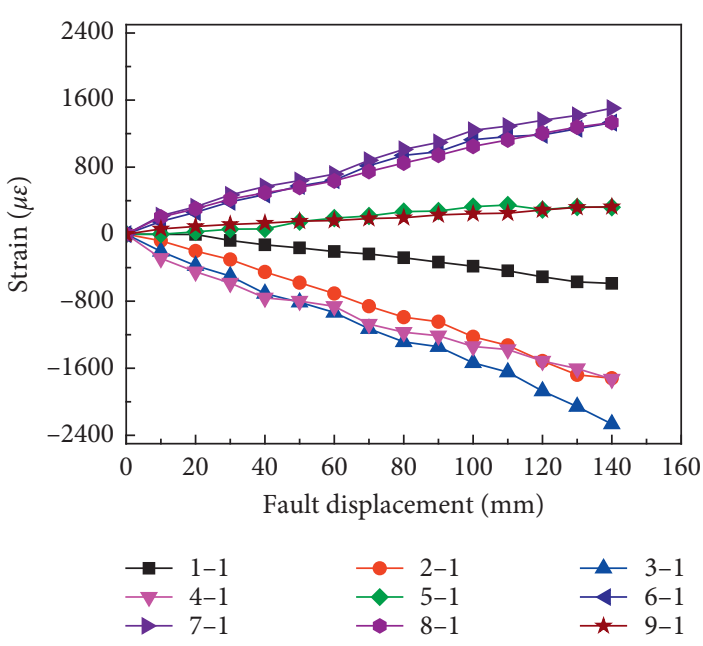

(a)

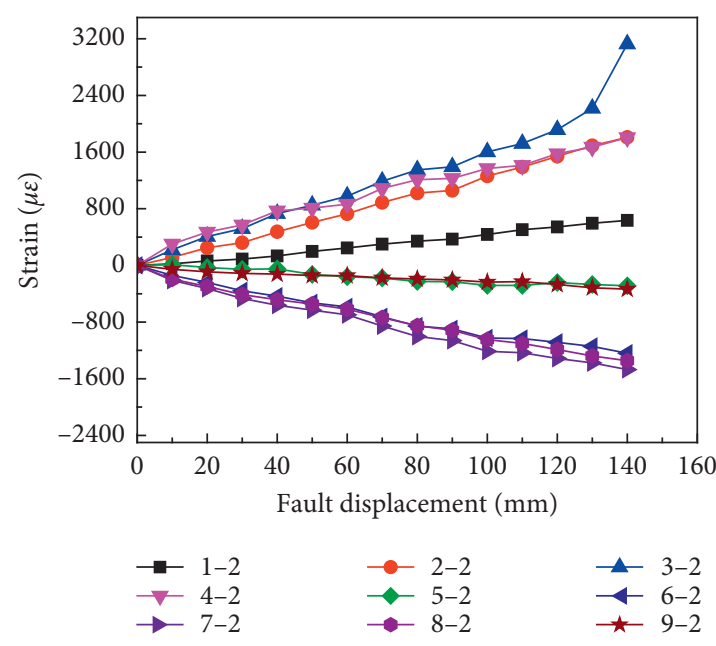

(b)

FIGURE 11: Strain-fault displacement curves of OC-6: (a) left-upper side; (b) right-under side.

change of axial strain in Figure 7(b). Due to the influence of the internal force change of the pipe section at the measuring point 5-1 on the adjacent pipe section, with the increase of the fault displacement, the axial compressive strain at the measuring points $(2-1,3-1$, and 4-1 in Figure 8) showed a trend of first increasing and then decreasing. Moreover, the axial tensile strain of the pipe section at measuring point 6-1 shows a sharp increase trend when the fault displacement is $60 \mathrm{~mm}$, while the axial compressive strain of the pipe section at measuring point 7-1 is relatively less affected; that is, the axial strain of the pipe section at each measuring point is affected by the adjacent pipe section and the distance between the pipe section to the fault at the measuring point. In the same way, the strain changes of the pipe section near the fault can be explained in Figures 9-11.
3.2. Effect of the Pipeline Diameters. Three types of pipelines with diameters of $25 \mathrm{~mm}, 32 \mathrm{~mm}$, and $40 \mathrm{~mm}$ were selected for mechanical properties tests, as shown in Figures 12(a)-12(f); the effect of different pipeline diameters on the strain of specimens under faults are studied. It can be found that when the fault displacement is $140 \mathrm{~mm}$, the strain response of pipelines with the larger diameter is relatively small, which can be attributed to the fact that increasing the diameter of the pipeline is equivalent to the increase of the moment of inertia of the cross-section, and thus the stiffness of the pipeline becomes larger. Therefore, the pipelines with a larger diameter should be chosen in the process of laying buried pipelines in practical projects, which is helpful to bear load and resist deformation. 


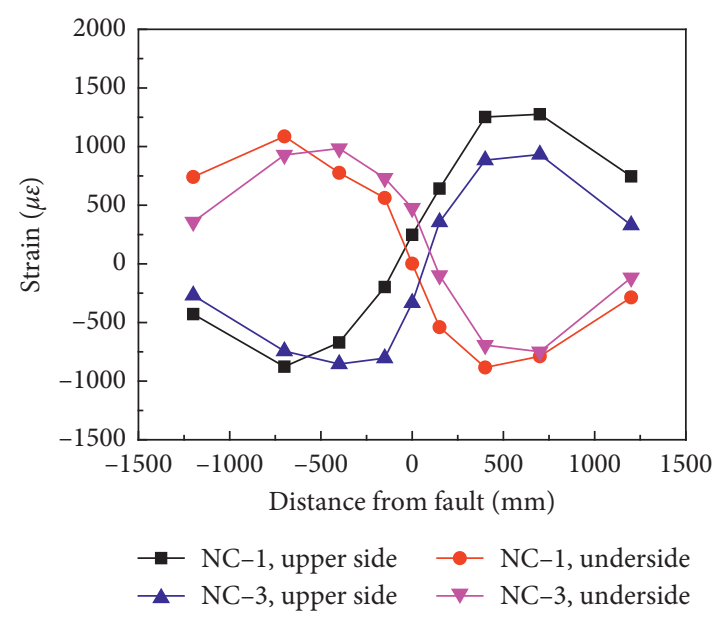

(a)

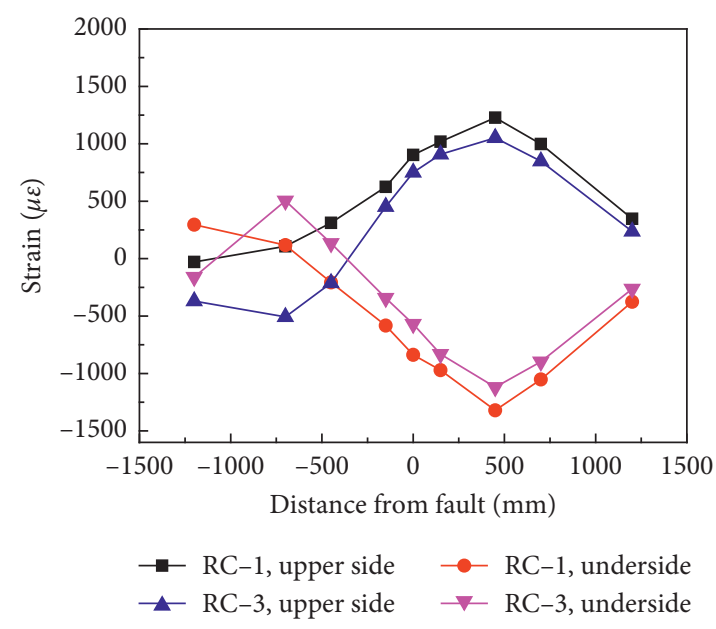

(c)

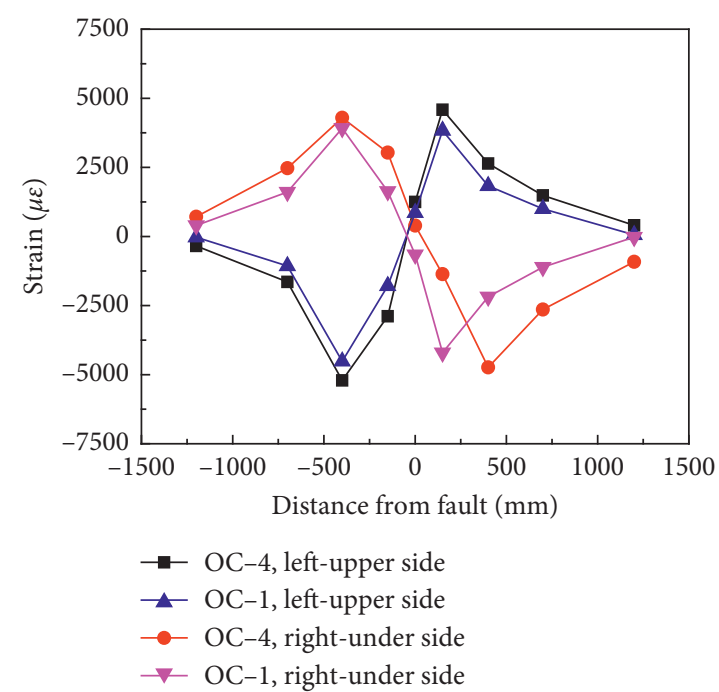

(e)

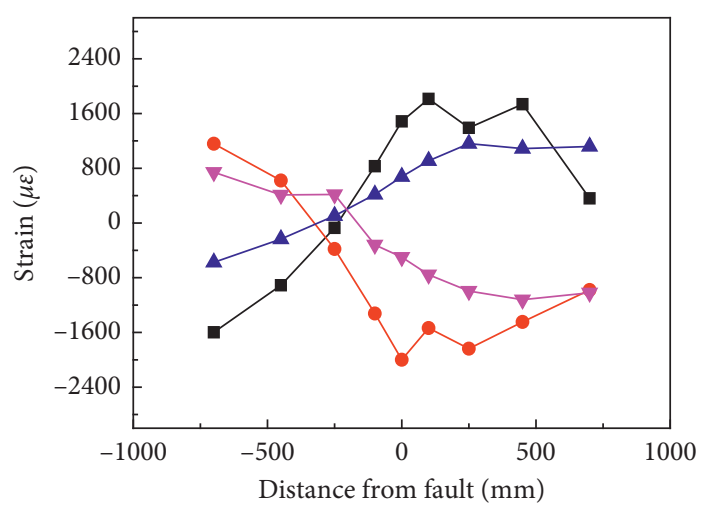

$\rightarrow$ NC-4, upper side $\longrightarrow$ NC-4, underside

$\neg$ NC-6, upper side $\rightarrow$ NC-6, underside

(b)

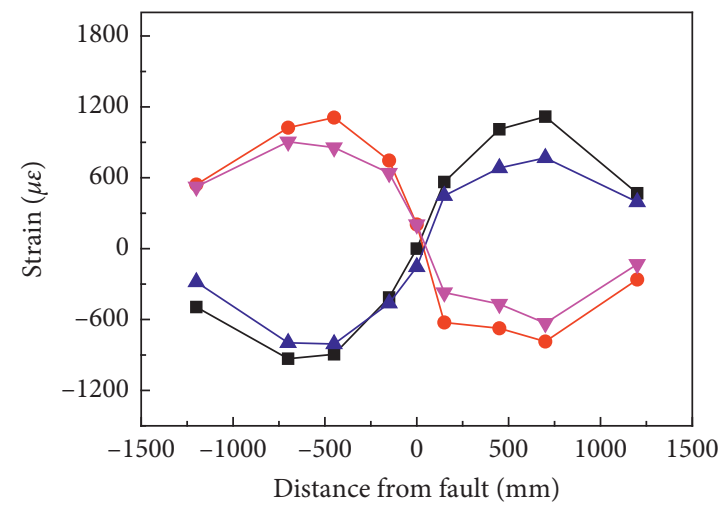

$\rightarrow$ SC-1, left side $\quad \rightarrow$ SC- 1 , right side
$\multimap$ SC-3, left side $\quad \rightarrow$ SC- 3 , right side

(d)

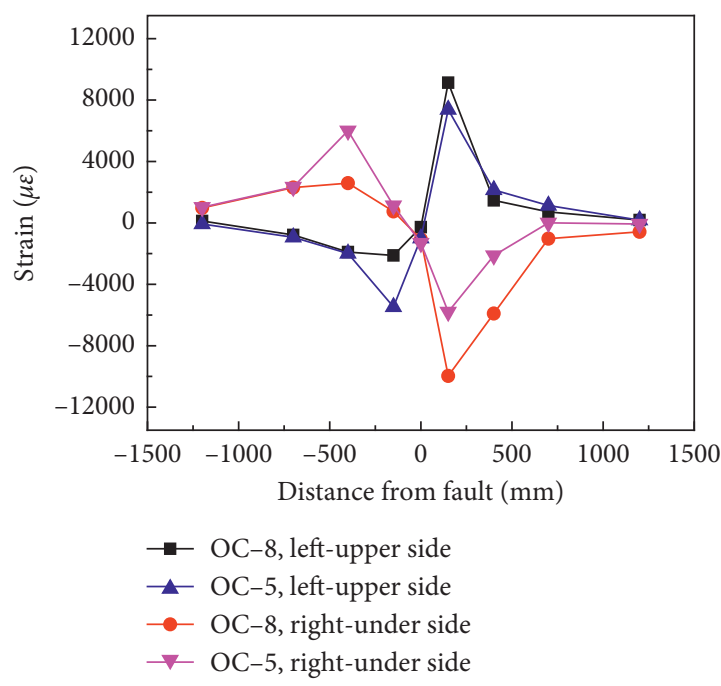

(f)

FIGURE 12: Effect of the pipeline diameters on the strain of specimens: (a) NC-1 and NC-3; (b) NC-4 and NC-6; (c) RC-1 and RC-3; (d) SC-1 and SC-3; (e) OC-1 and OC-4; (f) OC-5 and OC-8. 
3.3. Effect of the Wall Thicknesses. Two types of pipelines with wall thicknesses of $1.0 \mathrm{~mm}$ and $2.0 \mathrm{~mm}$ were selected for mechanical properties tests, as shown in Figures 13(a)13(c), which present the effect of the wall thicknesses on the strain of specimens. It can be seen that whether it is on the left-upper side or the right-under side of the pipelines, the strain response of pipelines with larger wall thickness is relatively small, and both the peak compressive strain and the peak tension strain occur near $500 \mathrm{~mm}$ from the fault. Thus, it shows that pipelines with larger wall thickness have better resistance to the fault movement.

3.4. Effect of the Buried Depths. Two types of pipelines with buried depths of $0.3 \mathrm{~m}$ and $0.6 \mathrm{~m}$ were selected for mechanical properties tests, as shown in Figures 14(a)-14(d), which present the effect of the buried depths on the strain of specimens. It can be seen that the strain responses of $\mathrm{NC}-1$, RC-1, SC-1, and OS-10 are slightly smaller than those of NC2, RC-2, SC-2, and OS-9, respectively, which indicates that the pipelines crossing faults should be shallowly buried. In addition, this indicates that the greater the buried depth of pipelines, the closer the position of the peak strain of tension and compression to the fault; the main reason is that the soil pressure acting on the pipelines is greater, and a small amount of energy produced by the fault movement is balanced by soil pressure, which results in the deformation area of the pipelines being closer to the fault area.

3.5. Effect of the Soil Materials. Two types of soil materials were selected to study the effect on the strain of pipelines, as shown in Figures 15(a)-15(c). It can be seen that the strain response of pipelines is relatively small when medium sand is used as a backfill. In terms of material properties, the cohesion of clay is much greater than that of medium sand, which results in that the restraint strength of clay to pipelines is significantly greater than that of medium sand to pipelines, and the friction between soil and pipeline is larger; thus, the soil has a greater pulling effect on pipelines. Therefore, noncohesive soil materials such as gravel, which usually has a smaller friction coefficient, should be used as a backfill in the process of pipelines laying.

3.6. Effect of the Spring Constraints. In order to study the effect of restraint strength at both ends of a pipeline on its strain response, in this section, two types of springs, which have a relatively small or large elastic coefficient, were selected to study their effect on the strain of pipelines. As shown in Figures 16(a) and 16(b), it can be found that the strain response of pipelines with smaller elastic coefficient is weaker; the reason is that the spring with a larger elastic coefficient has greater restraint strength on both ends of the pipelines, which makes the pipelines far away from the fault unable to expand better along the axis direction, thus resulting in an increase of strain values of the pipelines. Therefore, it is unreasonable to set two ends of the pipelines as fixed ends in the model test unless the length of the pipelines is long enough.

\section{Finite Element Verification and Analysis}

4.1. Details of the Finite Element Model. The finite element model was established by ABAQUS software, as shown in Figures $17(\mathrm{a})-17(\mathrm{~d})$. Considering the nonlinearity relationship of pipeline-soil materials and inconsistent displacement in the process of fault movement, the pipeline was modeled using the four-node shell element with reducedintegration (S4R) and the soil was simulated by the eightnode reduced-integration brick solid element (C3D8R). Considering the bond-slip effect of pipeline-soil, the contact pair method was used in the interaction module, mainly including two parts: normal action and tangential action. In this article, the hard contact was adopted in the normal action, and the Coulomb friction model was used to transfer the shear stress of the pipeline-soil surface in the tangential action. In addition, the spring unit was selected to establish the pipeline-end constraint. The spring element is defined by the connectors in the interaction module: first, define the properties of the connection section, select the axis for the translation type, select elastic for the behavior option, and define it as nonlinear. Finally, input the relationship data between force and displacement to complete the definition of spring properties; the second step is to create a connection pair: one end is connected to the node of the pipe end unit and the other end is connected to the reference point. The setting of the spring coefficient " $k$ " is simulated by specifying the spring coefficient in the connecting element. The specific parameters are shown in Table 1 . In the mesh generation module, the pipeline mesh within $800 \mathrm{~mm}$ from the fault side was compacted and seeded. The sensitivity analysis of the established mesh has been carried out, and the quality of the mesh has been optimized by continuously adjusting the topological relationship and node positions.

Considering the characteristics of the material, the threelinear model and the Mohr-Coulomb model were used to simulate the pipe and the soil $[25,26]$. For the setting of boundary conditions, the upper surface of the model is the ground surface, and there is no need to add boundary conditions. Under normal faults, displacement constraints and angle constraints in $X$-, $Y$-, and $Z$-directions are applied to the bottom surface of the fixed end, and corresponding displacement loads are applied to the bottom surface of the moving end. The front and posterior surfaces and the left and right surfaces are only constrained by displacement in the $X$-direction. Under strike-slip faults, displacement and angle constraints in $X$-, $Y$-, and $Z$-directions are applied to the bottom surface of the fixed end. The displacement constraints in the direction of $X$ and $Y$ are applied to the front and posterior surfaces of the fixed end, while the displacement constraints in the direction of $Y$ are applied only to the front and rear surfaces of the moving end. Under the oblique fault, the boundary conditions and displacement loads of normal faults and strike-slip faults need to be 


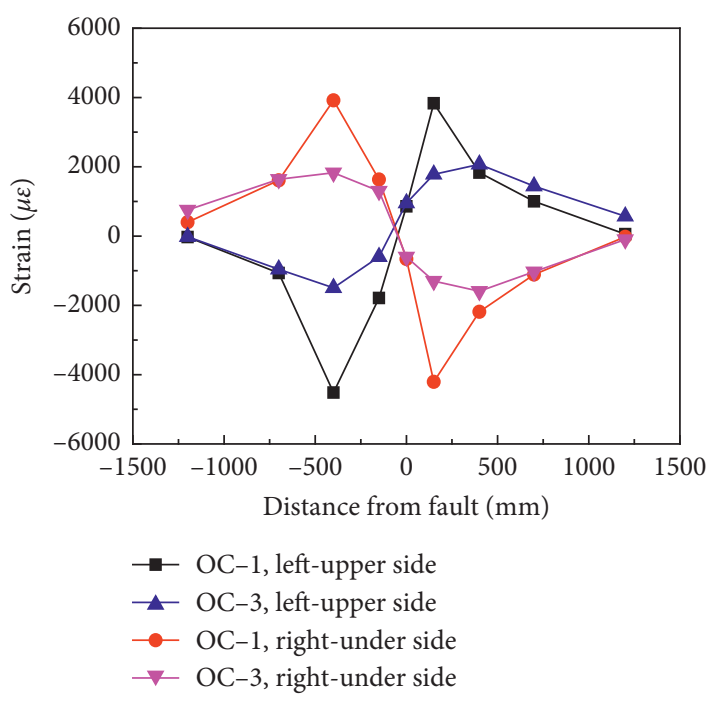

(a)

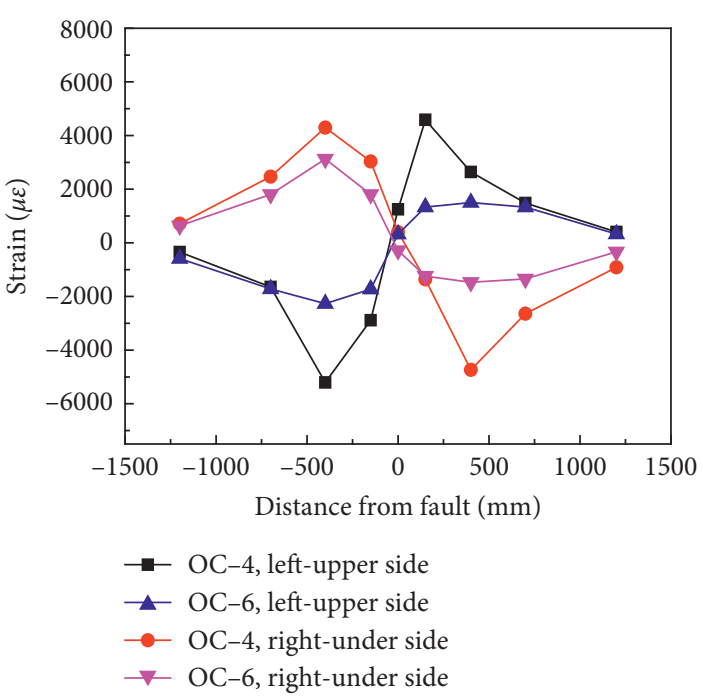

(b)

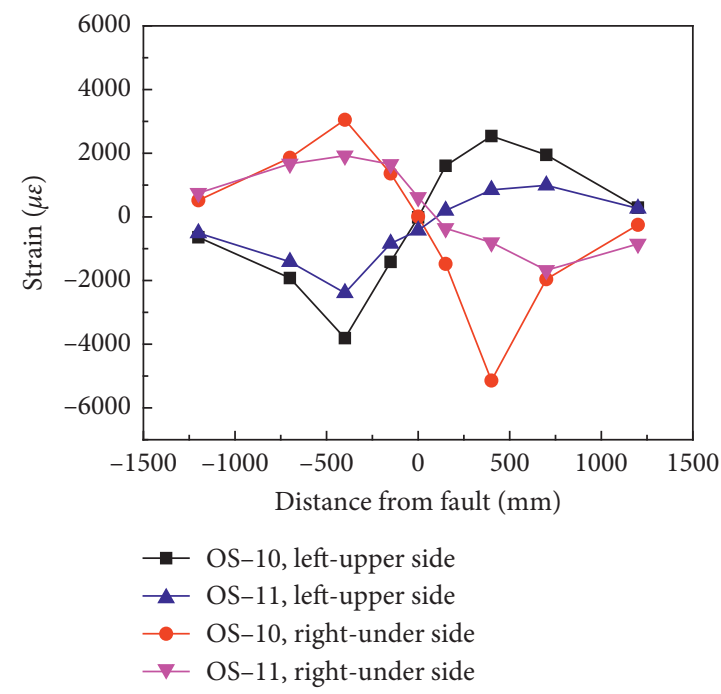

(c)

FIGURE 13: Effect of the wall thicknesses on the strain of specimens: (a) OC-1 and OC-3; (b) OC-4 and OC-6; (c) OS-10 and OS-11.

considered comprehensively. The displacement loading method is adopted for simulated loading; that is, displacement is applied to the bottom or side of the mobile terminal.

\subsection{The Relationship between Strain and Fault Displacement}

Time History. In order to investigate the dynamic effect of pipelines, an EI Centro seismic wave with a peak displacement of $140 \mathrm{~mm}$ was selected, as shown in Figure 18. The original acceleration of the EI Centro seismic wave was integrated twice, and the baseline was corrected. The obtained displacement curve was applied to the model soil for loading, and the boundary conditions did not change. When inputting seismic waves, taking into account the research of related scholars, the ground motion is scaled at a scale of 1 : 15. The strain of pipeline in the condition of EI Centro seismic wave was compared with the corresponding fault displacement, showing that there is a strong correlation between strain response time history and fault displacement time history. The pipeline strain of measuring point 7-1 of NC-5 changes with the fluctuation of displacement time history, and similar phenomena exist at other measuring points. Therefore, it can be considered that the vibration response of pipelines mainly depends on the inconsistent movement of soil at both ends of fault; a static test can be used to study the mechanical properties of pipelines under fault movement.

4.3. Equivalent Spring Boundary Verification. To verify the equivalent spring boundary proposed in this article, the calculation results of the fixed shell model were compared with the equivalent spring boundary model. The fixed shell model was set as follows: the diameter of the pipe was $32 \mathrm{~mm}$, the total length of the pipe was $60 \mathrm{~m}$, the soil is simulated with clay, the fault displacement was set to 


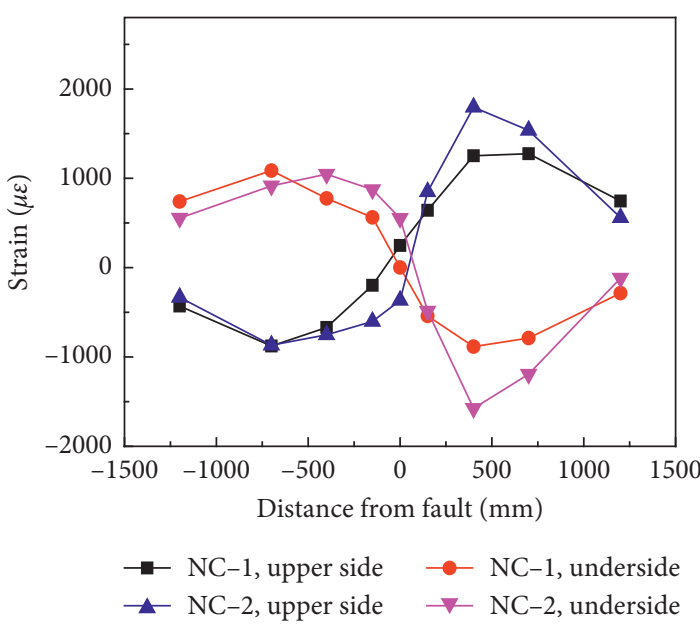

(a)

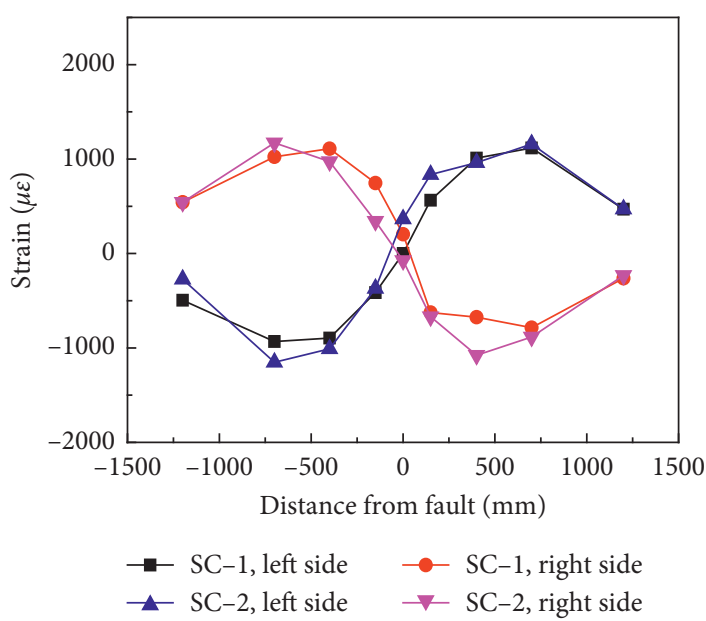

(c)

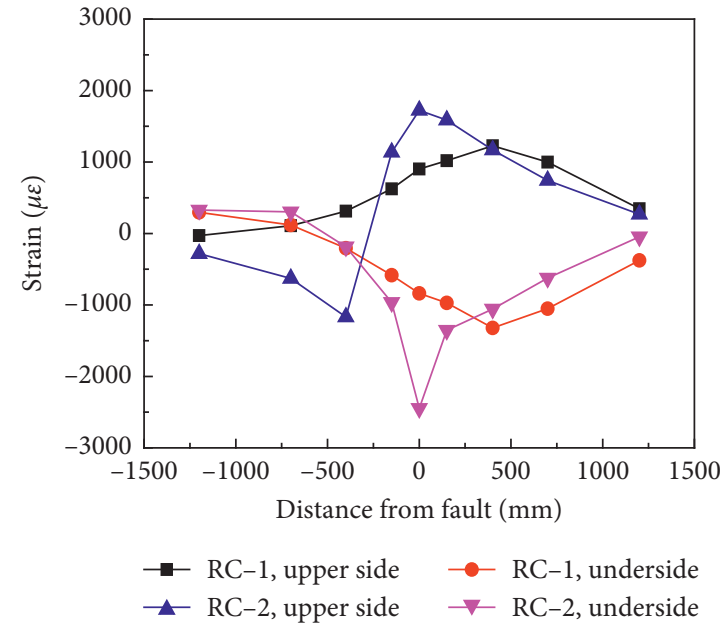

(b)

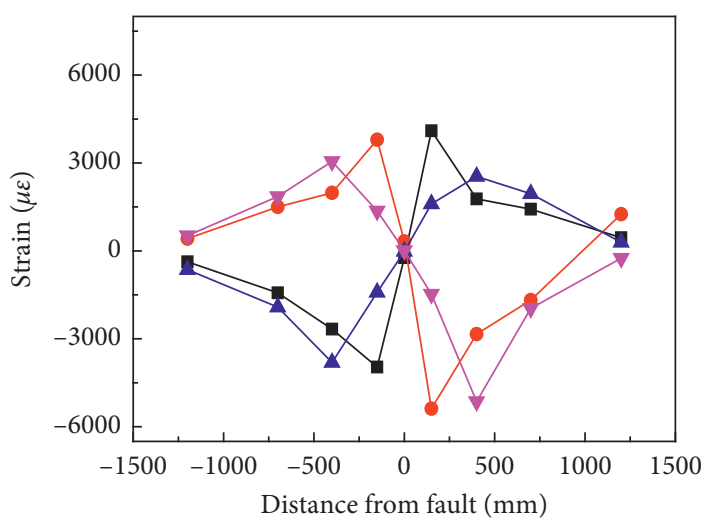

$\rightarrow$ OS-9, left-upper side

$\neg$ OS-10, left-upper side

- OS-9, right-under side

$\rightarrow$ OS-10, right-under side

(d)

FIGURE 14: Effect of the buried depths on the strain of specimens: (a) NC-1 and NC-2; (b) RC-1 and RC-2; (c) SC-1 and SC-2; (d) OS-9 and OS-10.

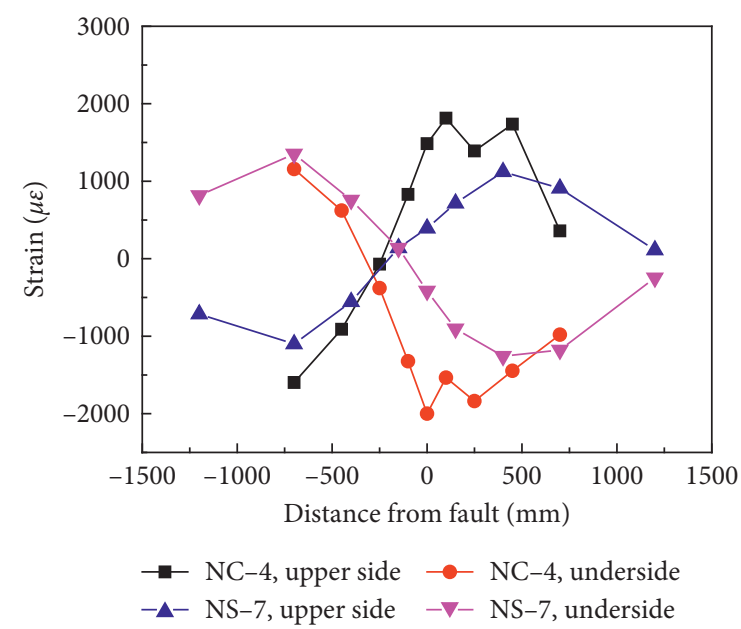

(a)

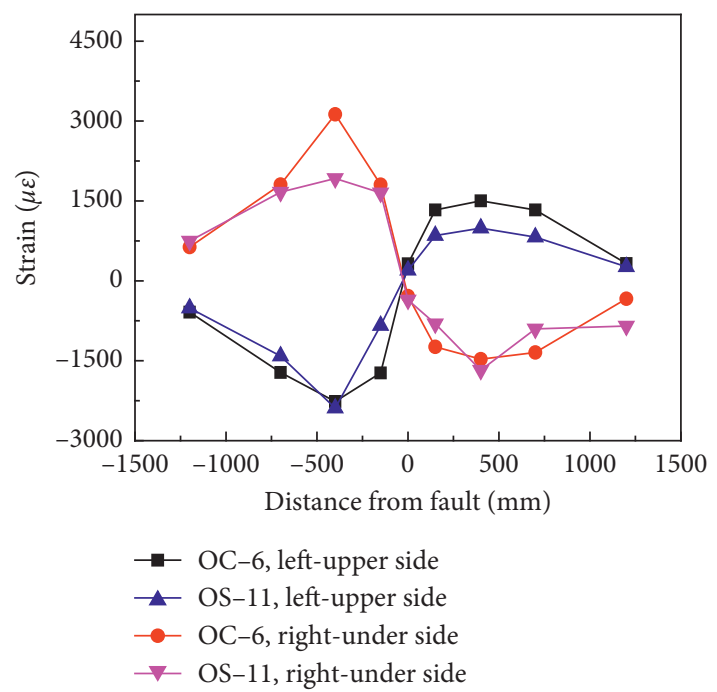

(b)

Figure 15: Continued. 


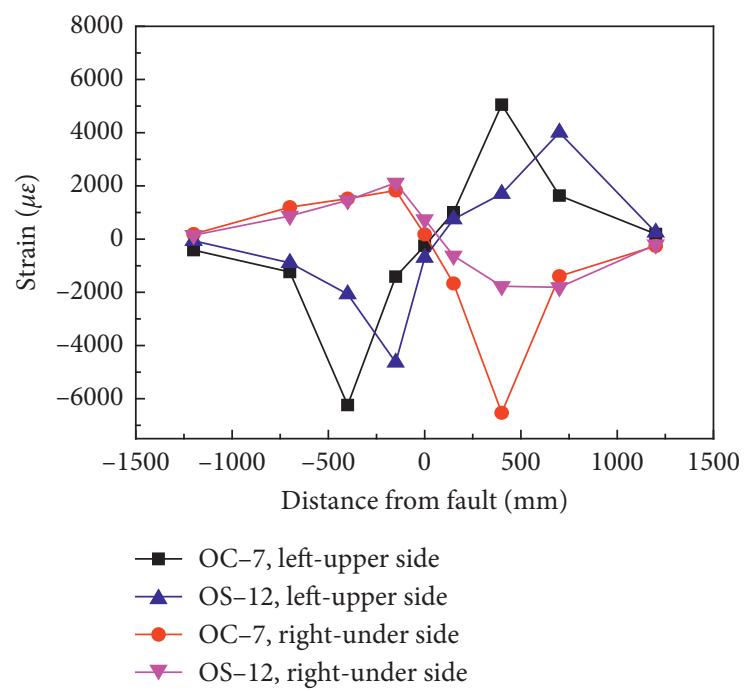

(c)

Figure 15: Effect of the soil materials on the strain of specimens: (a) NC-4 and NS-7; (b) OC-6 and OS-11; (c) OC-7 and OS-12.

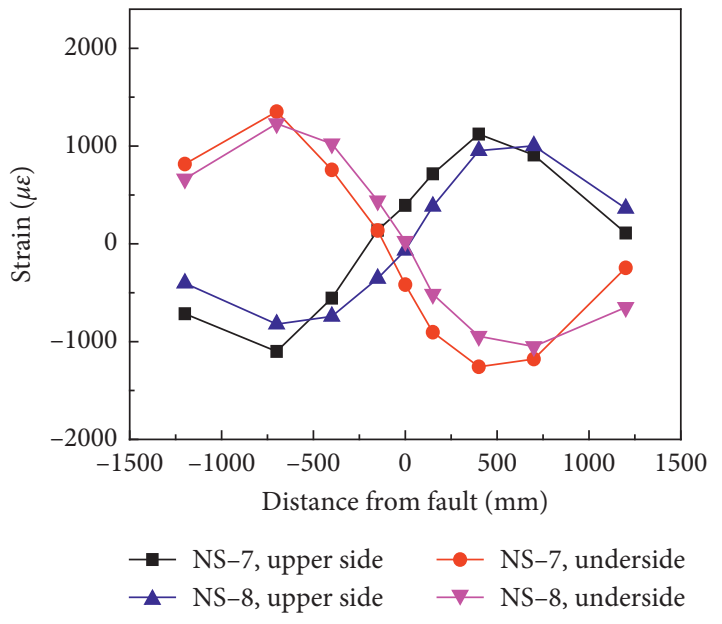

(a)

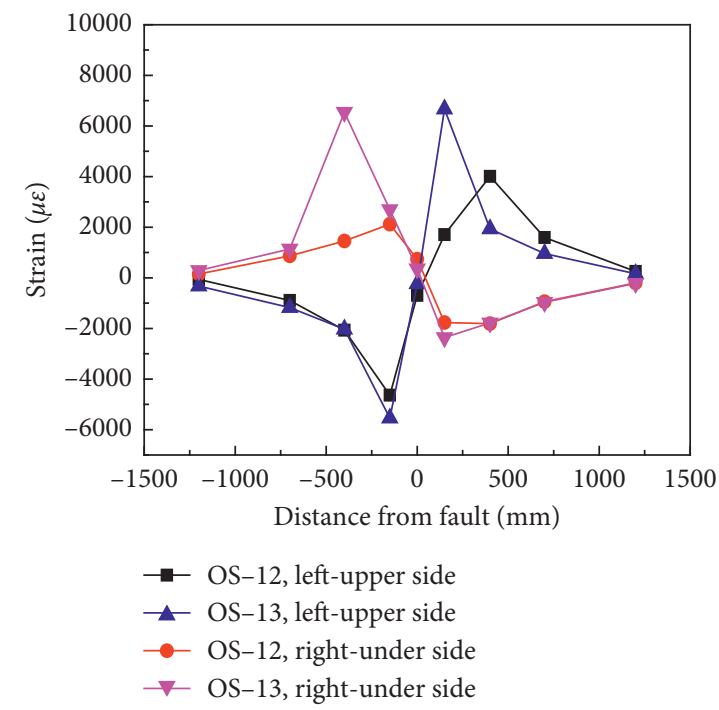

(b)

FIGURE 16: Effect of the spring constraints on the strain of specimens: (a) NS-7 and NS-8; (b) OS-12 and OS-13.

$140 \mathrm{~mm}$, the angle crossing normal fault was $90^{\circ}$, and the buried depth of the pipe was $0.3 \mathrm{~m}$. The only difference in the equivalent spring boundary model is that the total length of the pipe is $3.0 \mathrm{~m}$, and the other conditions are the same.

As shown in Figures 19(a) and 19(b), in which FSM and ESM stand for the fixed shell model and the equivalent spring model, respectively, it can be found that there is little difference between the Mises stress and the vertical deformation under these two cases. It is verified that the equivalent spring boundary model can better simulate the axial tension of the small deformation section of the pipeline and reduce the length of the pipeline required for the model test. Therefore, the effect of different parameters on the resistance to fault movement of pipeline can be studied through the attachment of equivalent spring boundary at both ends of the pipeline.

4.4. Comparison of Experimental Results and Finite Element Results. As shown in Figures 20(a)-20(f), when the fault displacement was $20 \mathrm{~mm}, 60 \mathrm{~mm}, 100 \mathrm{~mm}$, and $140 \mathrm{~mm}$, the axial strain and vertical deformation distribution along the tube axis of different specimens were compared in which FEM and Exp represented the finite element method and the experimental method, respectively. It can be seen that the finite element results are in good agreement with the experimental results, and the strain curve of the pipelines presents a centrosymmetric distribution. With the increase of fault displacement, the strain and deformation values of 


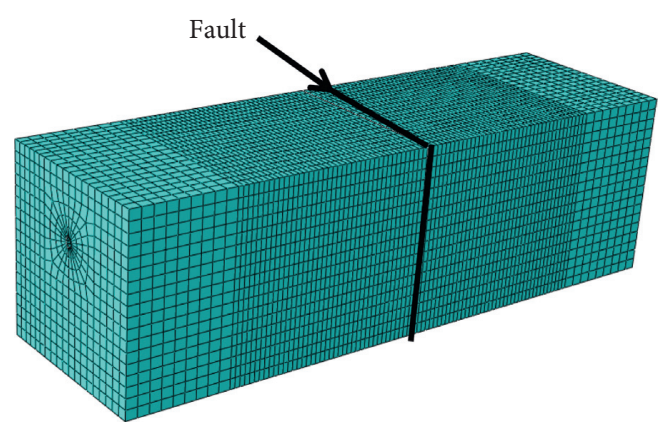

(a)

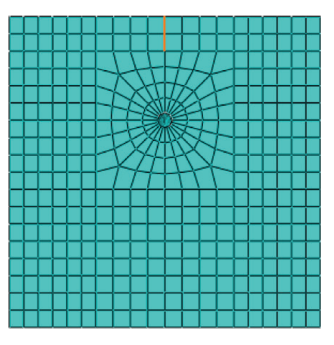

(b)

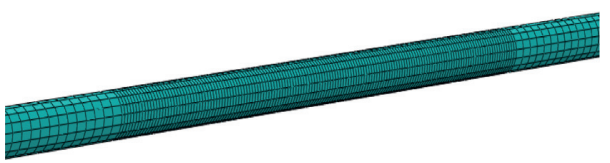

(c)

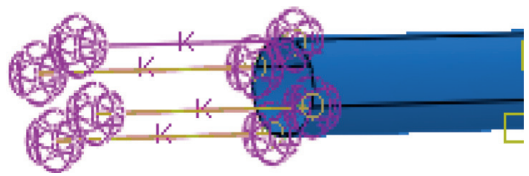

(d)

Figure 17: The finite element model: (a) integral mesh generation of soil; (b) mesh generation of soil section; (c) mesh generation of pipeline; (d) spring at pipeline end.

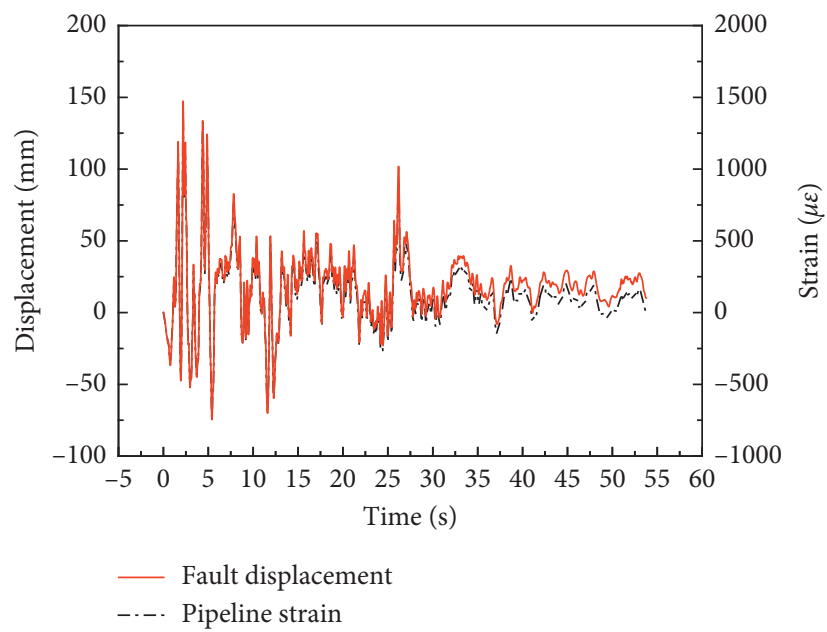

Figure 18: Time-history comparison of pipeline strain and fault displacement at point 7-1 of the NC-5 specimen.

pipelines increase. The strain and deformation response of pipelines under fault displacement can be well simulated based on the equivalent spring as a pipeline-end constraint.

\subsection{Analysis of Influence Parameters}

4.5.1. Effect of the Wall Thicknesses. Figures 21(a)-21(e) show the equivalent plastic strain (PEEQ) nephogram of pipelines with different wall thicknesses when the fault displacement was $2.0 \mathrm{~m}$. With the increase of wall thickness, the overall deformation of pipelines shows a weak trend, and the degree of stress concentration gradually weakens. Therefore, choosing pipelines with larger wall thickness is beneficial to prevent local buckling.

4.5.2. Effect of the Pressure. As shown in Figure 22, six different pipeline pressures, $0 \mathrm{MPa}, 4 \mathrm{MPa}, 6 \mathrm{MPa}, 8 \mathrm{MPa}$, $10 \mathrm{MPa}$, and $12 \mathrm{MPa}$, were selected as variables to study their effects on the strain response of pipelines. When the pipeline pressure is small, the effect of pressure on the peak strain of the pipelines is not obvious. Once the pipeline pressure is greater than $4 \mathrm{MPa}$, the peak tension and compression strain of the pipelines will increase rapidly. Therefore, the application of pressure is not conducive to the resistance of pipelines to fault movement.

4.5.3. Effect of the Soil Materials. Since the buried pipeline is laid in a wide range, the geological conditions and the stiffness of soil materials are important parameters affecting the mechanical properties of pipelines. Three representative soil materials, silty clay, medium sand, and hard clay, were selected for numerical simulation, as shown in Figure 23. It can be seen that the pipeline crossing in hard clay will enter the plastic strain concentration state more quickly. When 


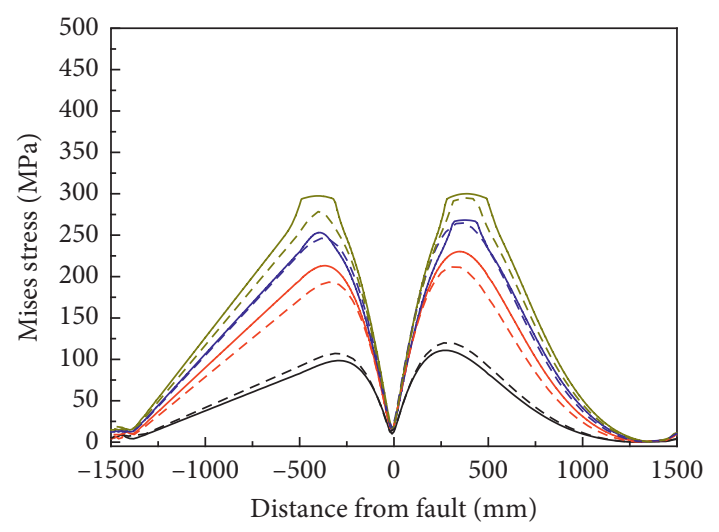

Fault displacement $(\mathrm{mm})$

$$
\begin{array}{ll}
-20(\mathrm{FSM}) & -60(\mathrm{FSM}) \\
-100(\mathrm{FSM}) & -140(\mathrm{FSM}) \\
---20(\mathrm{ESM}) & --60(\mathrm{ESM}) \\
---100(\mathrm{ESM}) & ---140(\mathrm{ESM})
\end{array}
$$

(a)

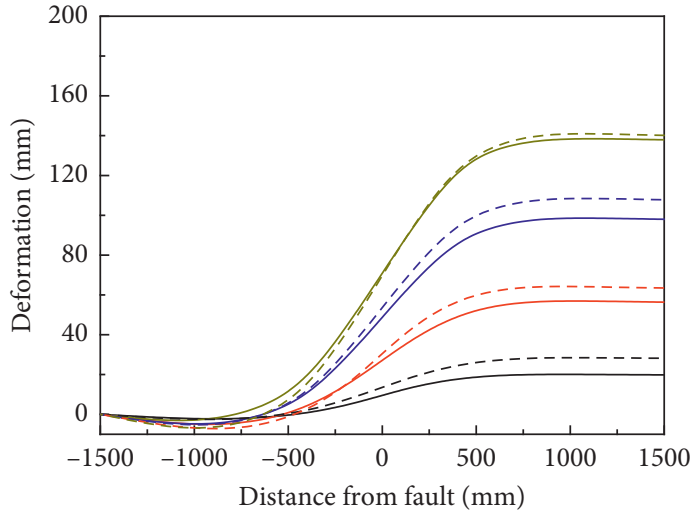

Fault displacement (mm)

$$
\begin{aligned}
& -20 \text { (FSM) } \\
& -100 \text { (FSM) } \\
& ---20(\text { ESM }) \\
& ---100 \text { (ESM) }
\end{aligned}
$$

(b)

FIgURE 19: Comparison of fixed shell model and equivalent spring model: (a) pipeline strain; (b) pipeline deformation.

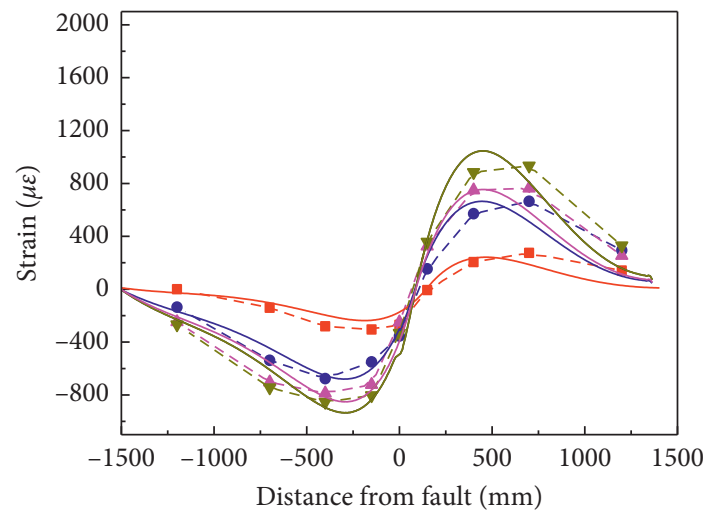

Fault displacement (mm)

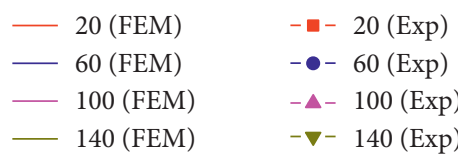

(a)

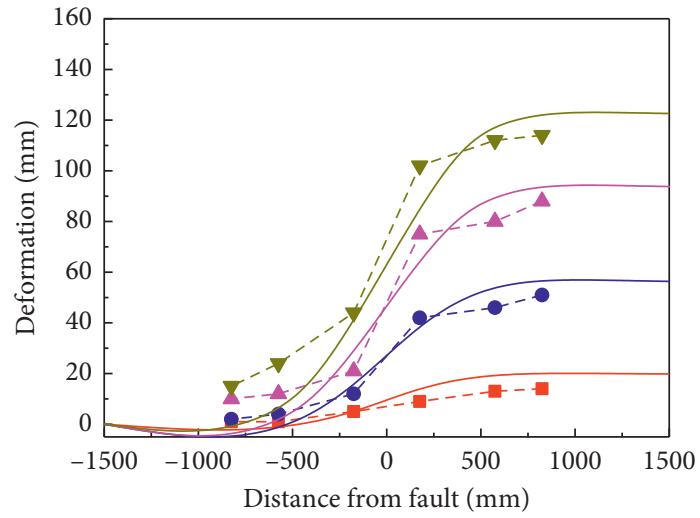

Fault displacement (mm)

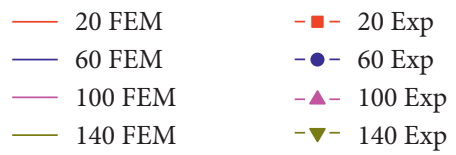

(b)

FIgURe 20: Continued. 

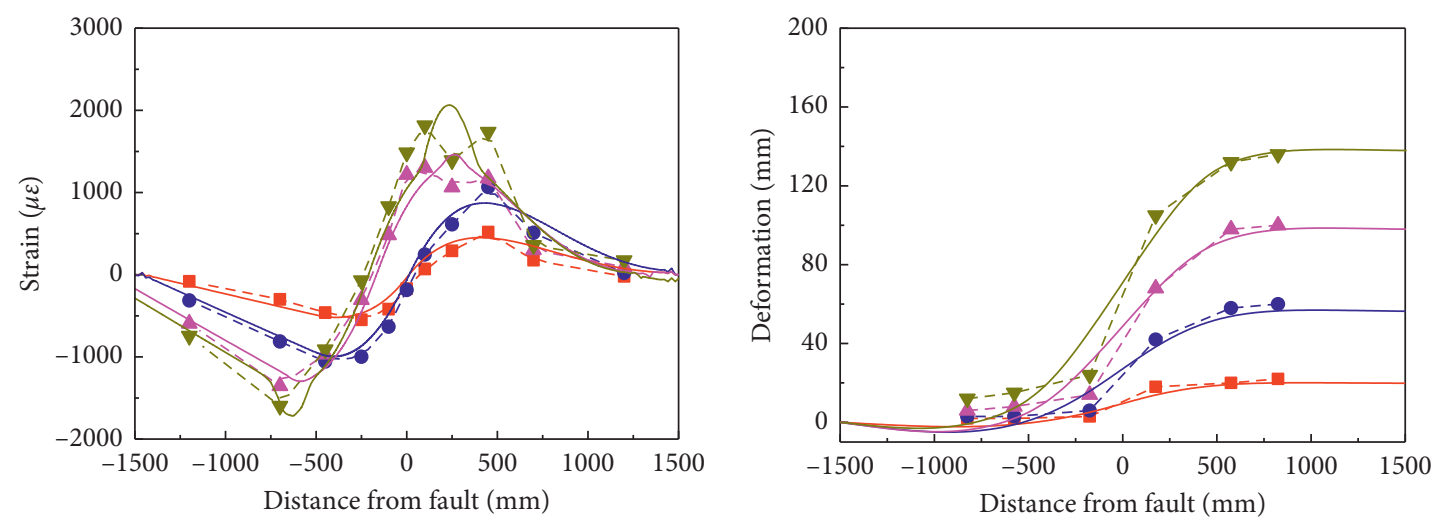

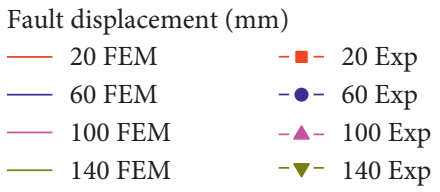

(c)

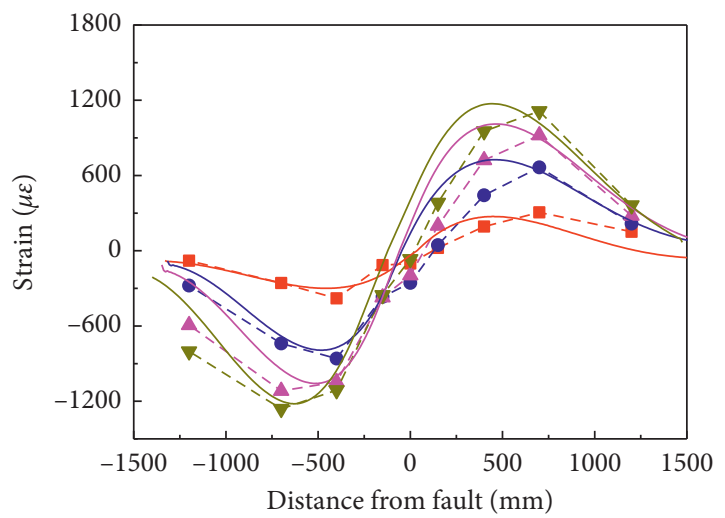

Fault displacement (mm)

$\begin{array}{ll}-20 \mathrm{FEM} & -\square \text { - } 20 \text { Exp } \\ -60 \mathrm{FEM} & -\bullet 60 \mathrm{Exp} \\ -100 \mathrm{FEM} & -\boldsymbol{-}-100 \mathrm{Exp} \\ -140 \mathrm{FEM} & -\nabla-140 \mathrm{Exp}\end{array}$

(e)

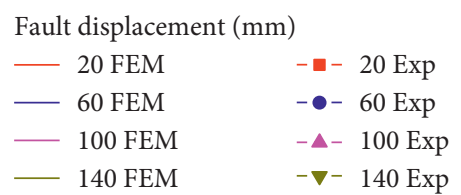

(d)

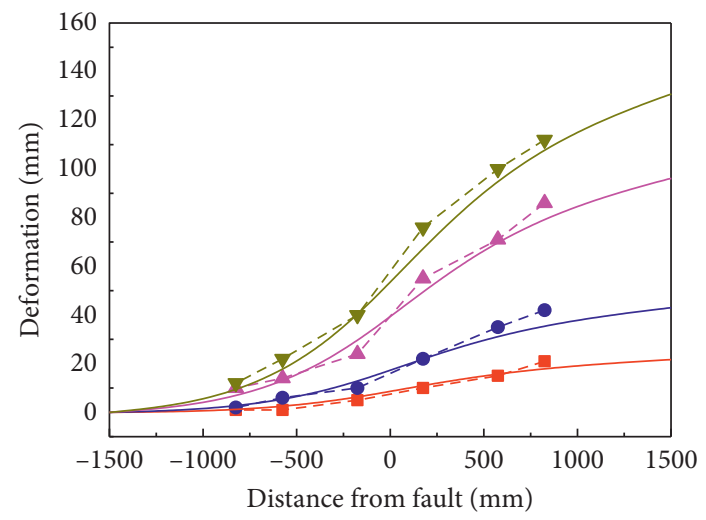

Fault displacement $(\mathrm{mm})$

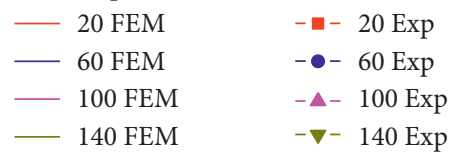

(f)

Figure 20: Comparison of experimental results and finite element results: (a) strain of NC-1; (b) deformation of NC-1; (c) strain of NC-4; (d) deformation of NC-4; (e) strain of NS-8; (f) deformation of NS-8.

the fault displacement reaches $1.6 \mathrm{~m}$, the strain of pipeline crossing in silty clay increases slowly, while the strain of pipeline crossing in the sand and hard clay increases rapidly.

4.6. Simplified Formula for Strain Calculation of Pipelines. Relevant scholars have studied the strain calculation methods of pipelines crossing normal, reverse, and strikeslip faults, but few studies have been done on pipelines crossing oblique-slip faults. Compared with the normal, reverse, and strike-slip faults, the effect of oblique-slip fault on the strain response of pipelines is more significant. In this article, the simplified formula of the axial compressive strain of buried pipelines across oblique-slip fault is obtained as follows based on normalizing 13 examples in Section 2.1 (it should be emphasized that this simplified formula is only applicable to the strain calculation and analysis of buried pipelines across oblique-slip fault in this test).

$$
\begin{aligned}
Y & =0.00114+0.00390 X^{3}, \\
\varepsilon_{\max } & =Y \cdot\left(\frac{H}{1.5}\right)^{0.1} \cdot\left(\frac{0.022}{t}\right)^{0.04} \cdot\left(\frac{1.219}{D}\right)^{0.08} \cdot\left(\frac{E_{s}}{20}\right)^{0.05}, \\
X & =\frac{\theta}{\alpha_{c}}
\end{aligned}
$$




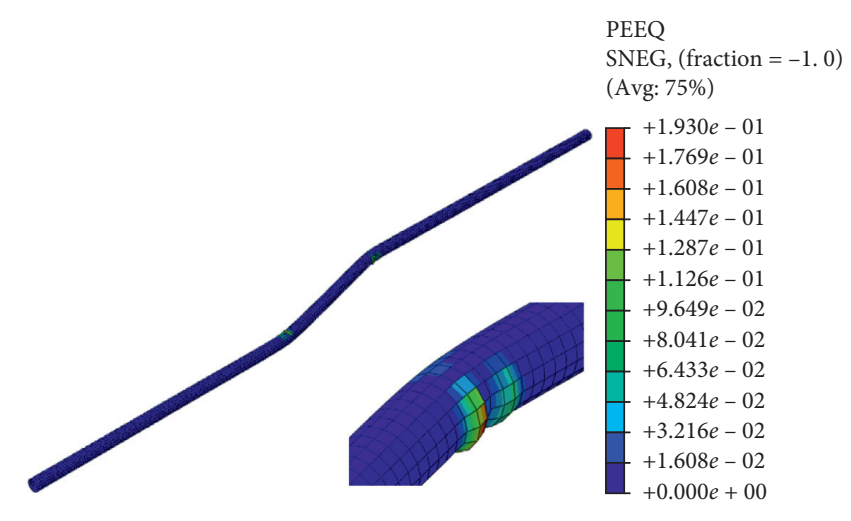

(a)

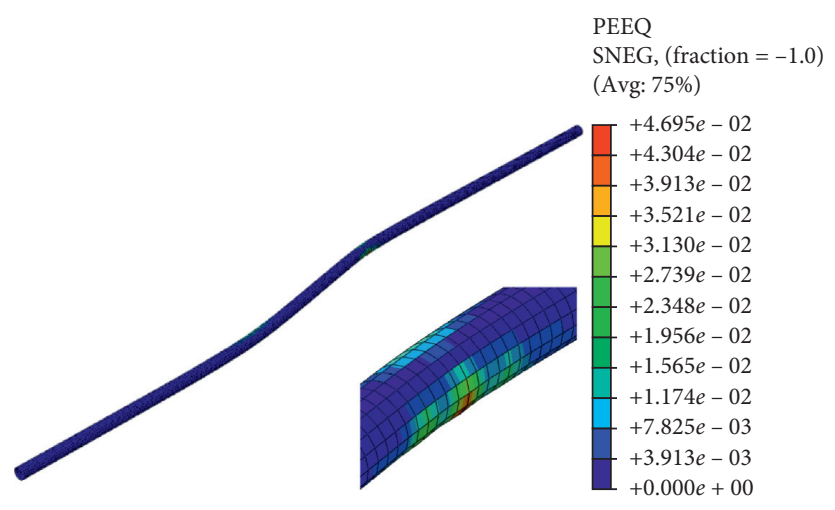

(c)

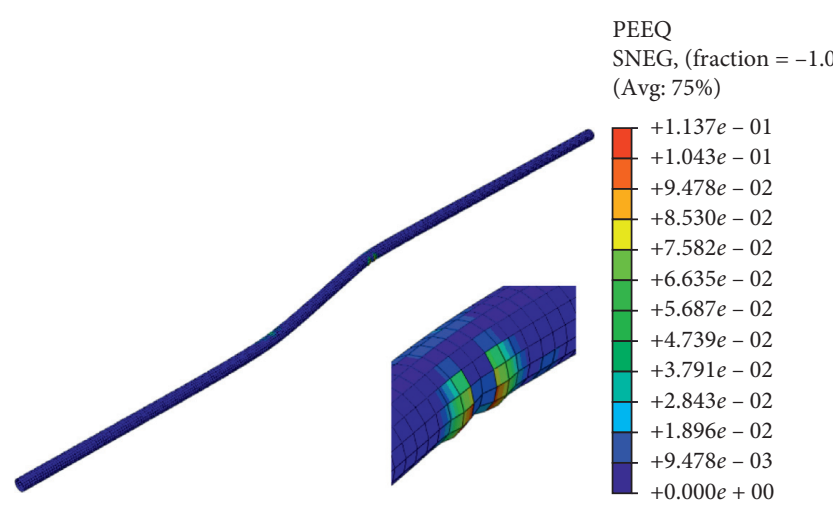

(b)

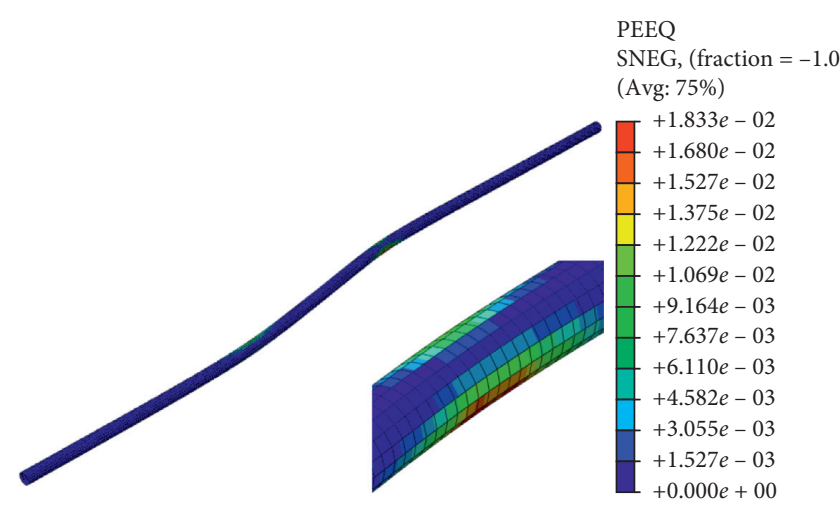

(d)

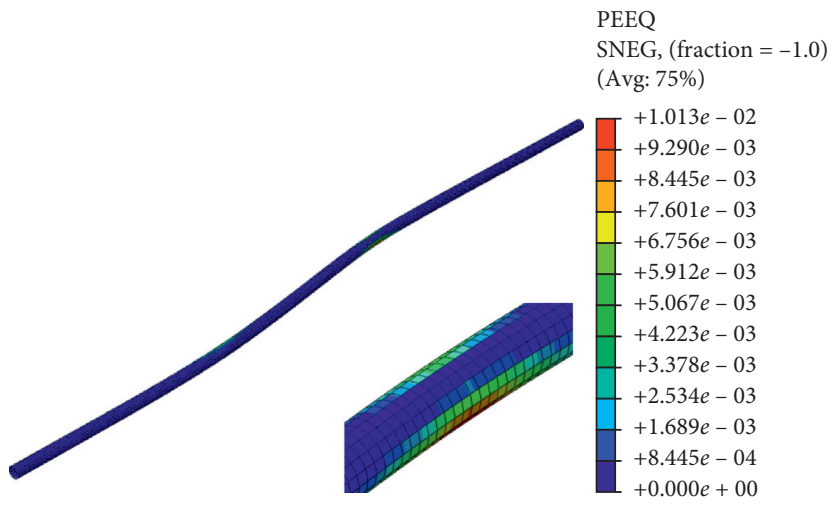

(e)

FIGURE 21: Equivalent plastic strain nephogram of pipelines: (a) $t=0.0153 \mathrm{~m}$; (b) $t=0.0184 \mathrm{~m}$; (c) $t=0.022 \mathrm{~m}$; (d) $t=0.0264 \mathrm{~m}$; (e) $t=0.033 \mathrm{~m}$.

where $(H / 1.5)^{0.1}$ represents the buried depth correction item of pipelines; $(0.022 / t)^{0.05}$ represents the wall thickness correction item of pipelines; $(1.219 / D)^{0.08}$ represents the pipeline diameter correction item; and $\left(E_{s} / 20\right)^{0.05}$ represents the elastic modulus correction item of soil materials.

For the buried pipeline model with a fault angle of $90^{\circ}$ in this article, the strain of the pipeline is dominated by compressive strain. The pipeline often produces buckling failure under the action of the fault. The buckling can be regarded as a plastic hinge, and the peak compressive strain of the pipeline is also related to the bending angle of the buckling. The bending angle $\theta$ can be expressed as follows:

$$
\begin{aligned}
\theta & =\arctan \frac{\Delta}{\sqrt{L_{p}^{2}+\Delta^{2}}}, \\
\alpha_{c} & =\frac{E / \sigma_{y}}{D / t} .
\end{aligned}
$$

where $L_{p}$ is the approximate length of the pipe with large deformation; $E$ is the elastic modulus of the pipe; $\sigma_{y}$ is the yield stress of the pipe; $D$ is the outer diameter of the pipe; $t$ is the pipe wall thickness; $\alpha_{c}$ is the dimensionless parameter related to the buckling deformation of the pipe. 


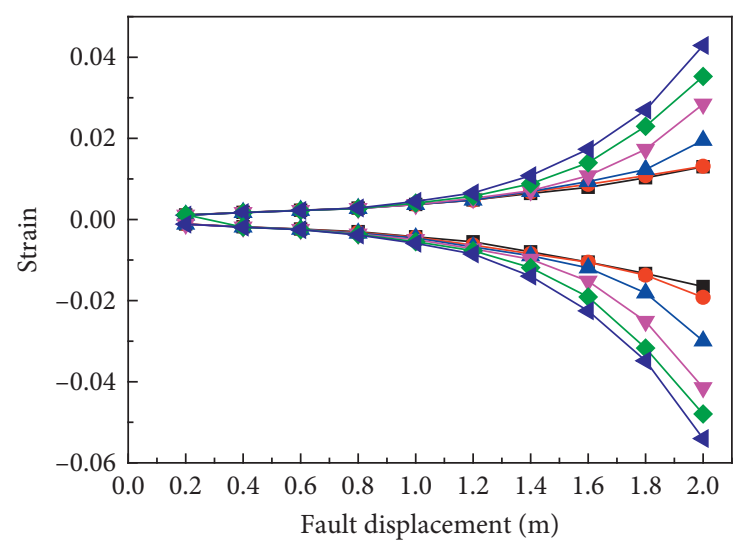

Pipeline pressure $(\mathrm{MPa})$

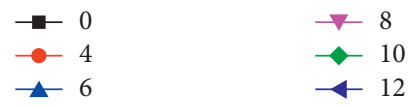

FIGURE 22: Relationship between strain and fault displacement under different pipeline pressures.

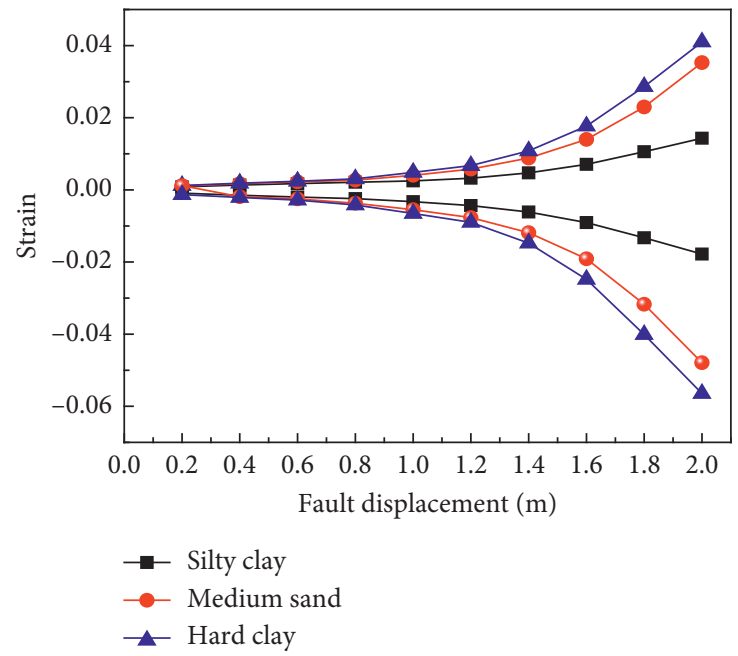

FIGURE 23: Relationship between strain and fault displacement under different soil materials.

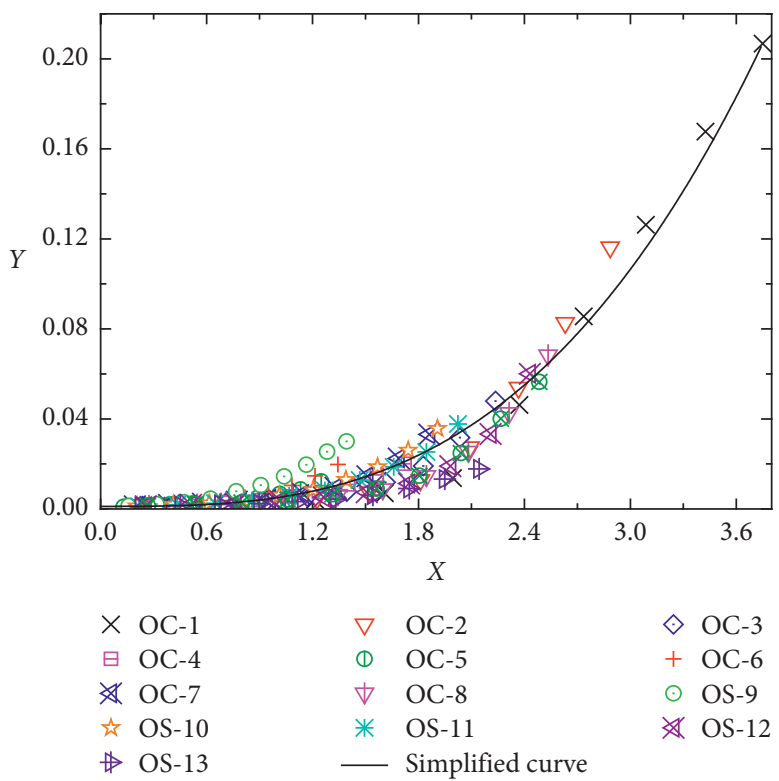

FIGURE 24: Simplified curve of pipeline strain calculation. 
Comparing the fitting curve with the example data, the goodness of fit is 0.9612 , as shown in Figure 24.

\section{Conclusions}

In this article, the mechanical behavior of buried pipelines subjected to faults is studied through experiments and numerical simulations. The following conclusions can be drawn:

(1) As the wall thickness and the diameter of the pipeline increase and the buried depth of the pipeline decreases, the peak strain of the buried pipeline decreases under the same working conditions. Therefore, increasing the wall thickness and pipe diameter and reducing the buried depth of the pipe are beneficial in reducing the strain response of the buried pipe.

(2) The two-end spring device can better control the size of the test model and enhance the reliability of the test results.

(3) The influence of pressure on the peak strain of the pipeline is not obvious when the pipeline pressure is small. However, when the pipeline pressure increases, the peak tensile and compressive strains of the pipeline will increase rapidly. At the same time, the less rigid soil material can delay the growth of pipeline strain.

(4) Based on the good agreement between the test results and the finite element analysis, the simplified formula of the axial compressive strain of buried pipelines across oblique-slip fault is obtained, which provides a reference for strain analysis of buried pipelines crossing oblique-slip faults.

\section{Discussion of Future Work}

In actual working conditions, pipelines may be damaged by corrosion and cracks [27-32]. Damage detection and health monitoring of the pipeline also receive much attention [33-35], and a variety of sensors, including fiber optical sensors [36-39] and piezoceramic transducers [40, 41], have been used for pipeline monitoring. In this study, we only consider health specimens, which is inconsistent with actual working conditions. One of the future studies will consider the analysis of damaged specimens and study how this damage occurs and the actual mechanism of action on the pipeline.

Detecting the damage of buried pipelines under fault movement in engineering is of great significance. Previous studies have shown that piezoceramic materials have good advantages in pipeline damage detection [42-44]. Thus, piezoceramic materials are commonly used in stress-wave or guided-wave-based damage detection [45-47] and health monitoring [48-50]. The PZT (Lead Zirconate Titanate), a type of piezoceramic material with a strong piezoelectric effect, is commonly used in damage detection of many different systems [51-53], including pipelines [54, 55]. However, the real-time damage detection of the buried pipeline during fault movement using PZT transducers has not been adequately addressed in the literature. In another future work, we will explore the real-time damage detection of a buried pipeline during fault movement using distributed PZT transducers and the time reversal method by taking advantage of the developed fault motion simulation experimental setup.

\section{Data Availability}

The data used to support the findings of this study are available from the corresponding author upon request.

\section{Conflicts of Interest}

The authors declare no conflicts of interest.

\section{Authors' Contributions}

All authors discussed and agreed upon the idea and made scientific contributions. Xiaolong Wei, Xi Zeng, and Guofeng $\mathrm{Du}$ designed the experiments and wrote the manuscript. Wenshuai Jiao and Danfu Zhang performed the experiments and analyzed the data.

\section{Acknowledgments}

The authors acknowledge the help of Xi Zeng from the Second Construction Engineering Co., Ltd., China Construction Third Engineering Bureau. This work was partially supported by Hubei Province Technical Innovation Major Special Project (Grant no. 2019AAA011), the National Natural Science Foundation of China (Grant no. 51778064), and the Scientific Research Project Foundation of Hubei Provincial Department of Education (Grant no. 2016CFA022).

\section{References}

[1] J. P. Bardet and C. A. Davis, "Lower San Fernando corrugated metal pipe failure," in Proceedings of the 1995 Conference on Lifeline Earthquake Engineering, San Francisco, CA, USA, August 1995.

[2] D. F. Palmer and T. L. Henyey, "San Fernando earthquake of 9 February 1971: pattern of faulting," Science, vol. 172, no. 3984, pp. 712-715, 1971.

[3] M. H. Erami, M. Miyajima, S. Kaneko, T. Toshima, and S. Kishi, "Pipe-soil interaction for segmented buried pipelines subjected to dip faults," Earthquake Engineering \& Structural Dynamics, vol. 44, no. 3, pp. 403-417, 2015.

[4] Q. M. Feng, E. D. Guo, Y. M. Song, H. Y. Gao, P. Z. Zhang, and W. D. Xu, "Aseismic test of buried pipe crossing fault," Earthquake Engineering and Engineering Vibration, vol. 20, no. 1, pp. 56-62, 2000.

[5] H. E. Demirci, S. Bhattacharya, D. Karamitros, and N. Alexander, "Experimental and numerical modelling of buried pipelines crossing reverse faults," Soil Dynamics and Earthquake Engineering, vol. 114, pp. 198-214, 2018.

[6] X. Zhang, X. H. Bai, and W. B. He, "The experiment of deformation and internal force of soft pipeline caused by subsoil settlement," Applied Mechanics and Materials, vol. 275-277, pp. 1493-1498, 2013. 
[7] Z. C. Zhang, J. T. Wang, and Y. J. Xu, "Shaking table test for cross-fault buried pipelines (I)-model design," China Civil Engineering Journal, vol. 44, no. 11, pp. 93-98, 2011.

[8] Z. C. Zhang, J. T. Wang, and Y. J. Xu, "Shaking table test for cross-fault buried pipelines (II)-test results," China Civil Engineering Journal, vol. 44, no. 12, pp. 116-125, 2011.

[9] D. F. Zhang, X. M. Bie, X. Zeng, Z. Lei, and G. F. Du, “An experimental and numerical studies on mechanical behavior of buried pipelines crossing faults," Structural Engineering and Mechanics, vol. 75, pp. 71-86, 2020.

[10] A. P. Tang, L. F. Wang, B. C. Wu, and M. J. O’Rourke, "Centrifuge model test on a buried pipeline crossing reverse fault considering soil-structure interactions," China Earthquake Engineering Journal, vol. 37, no. 3, pp. 639-642, 2015.

[11] L. Y. Li, C. B. Wang, J. Y. Han, B. W. Hou, C. A. Xu, and X. L. Du, "Analysis of site responses during shaking table test for the interaction between pipeline and soil," Earthquake Engineering and Engineering Dynamics, vol. 35, no. 3, pp. 166-176, 2015.

[12] A. V. Oskouei, A. Tamjidi, and P. Pourshabani, "Effects of burial depth in the behavior of buried steel pipelines subjected to strike-slip fault," Soil Dynamics and Earthquake Engineering, vol. 123, pp. 252-264, 2019.

[13] M. O’Rourke, V. Gadicherla, and T. Abdoun, "Centrifuge modeling of PGD response of buried pipe," Earthquake Engineering and Engineering Vibration, vol. 4, no. 1, pp. 6973, 2005.

[14] Y. W. Choo, T. H. Abdoun, M. J. O'Rourke, and D. Ha, "Remediation for buried pipeline systems under permanent ground deformation," Soil Dynamics and Earthquake Engineering, vol. 27, no. 12, pp. 1043-1055, 2007.

[15] D. Ha, T. H. Abdoun, M. J. O’Rourke et al., "Buried highdensity polyethylene pipelines subjected to normal and strikeslip faulting-a centrifuge investigation," Canadian Geotechnical Journal, vol. 45, no. 12, pp. 1733-1742, 2008.

[16] D. Ha, T. H. Abdoun, M. J. O’Rourke et al., “Centrifuge modeling of earthquake effects on buried high-density polyethylene (HDPE) pipelines crossing fault zones," Journal of Geotechnical and Geoenvironmental Engineering, vol. 134, no. 10, pp. 1501-1515, 2008.

[17] T. H. Abdoun, D. Ha, M. J. O’Rourke et al., "Factors influencing the behavior of buried pipelines subjected to earthquake faulting," Soil Dynamics and Earthquake Engineering, vol. 29, no. 3, pp. 415-427, 2009.

[18] J. Kim, S. S. Nadukuru, M. Pour-Ghaz et al., "Assessment of the behavior of buried concrete pipelines subjected to ground rupture: experimental study," Journal of Pipeline Systems Engineering and Practice, vol. 3, no. 1, pp. 8-16, 2012.

[19] M. Rojhani, M. Moradi, A. Galandarzadeh, and S. Takada, "Centrifuge modeling of buried continuous pipelines subjected to reverse faulting," Canadian Geotechnical Journal, vol. 49, no. 6, pp. 659-670, 2012.

[20] M. Moradi, M. Rojhani, A. Galandarzadeh et al., "Centrifuge modeling of buried continuous pipelines subjected to normal faulting," Earthquake Engineering and Engineering Vibration, vol. 12, no. 1, pp. 155-164, 2013.

[21] W. W. Sim, I. Towhata, S. Yamada, and G. J.-M. Moinet, "Shaking table tests modelling small diameter pipes crossing a vertical fault," Soil Dynamics and Earthquake Engineering, vol. 35, pp. 59-71, 2012.

[22] F. R. Rofooei, H. H. Jalali, N. K. A. Attari, and M. Alavi, "Fullscale laboratory testing of buried pipelines subjected to permanent ground displacement caused by reverse faulting," in Proceedings of the 15th World Conference on Earthquake Engineering, Lisbon, Portugal, September 2012.

[23] F. R. Rofooei, H. H. Jalali, N. K. A. Attari, H. Kenarangi, and M. Samadian, "Parametric study of buried steel and high density polyethylene gas pipelines due to oblique-reverse faulting," Canadian Journal of Civil Engineering, vol. 42, no. 3 , pp. 178-189, 2015.

[24] X. Zeng, F.-F. Dong, X.-D. Xie, and G.-F. Du, “A new analytical method of strain and deformation of pipeline under fault movement," International Journal of Pressure Vessels and Piping, vol. 172, pp. 199-211, 2019.

[25] J.-P. Tian, J. Zhang, F.-F. Dong, and G.-F. Du, "Dynamic response of buried pipeline subject to impact loads using piezoceramic transducers," International Journal of Pressure Vessels and Piping, vol. 177, Article ID 103984, 2019.

[26] F. Dong, X. Bie, J. Tian, X. Xie, and G. Du, "Experimental and numerical study on the strain behavior of buried pipelines subjected to an impact load," Applied Sciences, vol. 9, no. 16, p. 3284, 2019.

[27] Y. M. Zhang, D. K. Yi, Z. M. Xiao, Z. H. Huang, and S. B. Kumar, "Elastic-plastic fracture analyses for pipeline girth welds with $3 \mathrm{D}$ semi-elliptical surface cracks subjected to large plastic bending," International Journal of Pressure Vessels and Piping, vol. 105-106, no. 5, pp. 90-102, 2013.

[28] D. Arafah, M. Madia, U. Zerbst, S. Beretta, and M. E. Cristea, "Instability analysis of pressurized pipes with longitudinal surface cracks," International Journal of Pressure Vessels and Piping, vol. 126-127, pp. 48-57, 2015.

[29] X. Li, Y. Bai, C. Su, and M. Li, "Effect of interaction between corrosion defects on failure pressure of thin wall steel pipeline," International Journal of Pressure Vessels and Piping, vol. 138, pp. 8-18, 2016.

[30] H. A. Kishawy and H. A. Gabbar, "Review of pipeline integrity management practices," International Journal of Pressure Vessels and Piping, vol. 87, no. 7, pp. 373-380, 2010.

[31] G. Du, Q. Kong, F. Wu, J. Ruan, and G. Song, “An experimental feasibility study of pipeline corrosion pit detection using a piezoceramic time reversal mirror," Smart Materials and Structures, vol. 25, no. 3, Article ID 037002, 2016.

[32] G. Du, Q. Kong, T. Lai, and G. Song, "Feasibility study on crack detection of pipelines using piezoceramic transducers," International Journal of Distributed Sensor Networks, vol. 2013, Article ID 631715, 7 pages, 2013.

[33] G. Zhang, W. Gao, G. Song, and Y. Song, "An imaging algorithm for damage detection with dispersion compensation using piezoceramic induced lamb waves," Smart Materials and Structures, vol. 26, no. 2, Article ID 025017, 2017.

[34] H. Xiao, J. Zheng, and G. Song, "Severity evaluation of the transverse crack in a cylindrical part using a PZT wafer based on an interval energy approach," Smart Materials and Structures, vol. 25, no. 3, Article ID 035021, 2016.

[35] D. Ozevin and J. Harding, "Novel leak localization in pressurized pipeline networks using acoustic emission and geometric connectivity," International Journal of Pressure Vessels and Piping, vol. 92, pp. 63-69, 2012.

[36] Z. G. Jia, L. Ren, H. N. Li, W. Wu, and J. Tao, "Performance study of FBG hoop strain sensor for pipeline leak detection and localization," Journal of Aerospace Engineering, vol. 31, no. 5, 2018.

[37] L. Ren, Z.-G. Jia, H.-N. Li, and G. Song, "Design and experimental study on FBG hoop-strain sensor in pipeline monitoring," Optical Fiber Technology, vol. 20, no. 1, pp. 15-23, 2014. 
[38] Q. Hou, W. Jiao, L. Ren, H. Cao, and G. Song, "Experimental study of leakage detection of natural gas pipeline using FBG based strain sensor and least square support vector machine," Journal of Loss Prevention in the Process Industries, vol. 32, pp. 144-151, 2014.

[39] Z.-G. Jia, L. Ren, H.-N. Li, S.-C. Ho, and G.-B. Song, "Experimental study of pipeline leak detection based on hoop strain measurement," Structural Control and Health Monitoring, vol. 22, no. 5, pp. 799-812, 2015.

[40] G. Du, L. Huo, Q. Kong, and G. Song, "Damage detection of pipeline multiple cracks using piezoceramic transducers," Journal of Vibroengineering, vol. 18, no. 5, pp. 2828-2838, 2016.

[41] G. Du, Q. Kong, H. Zhou, and H. Gu, "Multiple cracks detection in pipeline using damage index matrix based on piezoceramic transducer-enabled stress wave propagation," Sensors, vol. 17, no. 8, p. 1812, 2017.

[42] M. Quant, H. Elizalde, A. Flores, R. Ramírez, P. Orta, and G. Song, "A comprehensive model for piezoceramic actuators: modelling, validation and application," Smart Materials and Structures, vol. 18, no. 12, Article ID 125011, 2009.

[43] B. N. Agrawal, M. A. Elshafei, and G. Song, "Adaptive antenna shape control using piezoelectric actuators," Acta Astronautica, vol. 40, no. 11, pp. 821-826, 1997.

[44] F. Wang, S. C. M. Ho, L. Huo, and G. Song, "A novel fractal contact-electromechanical impedance model for quantitative monitoring of bolted joint looseness," IEEE Access, vol. 6, pp. 40212-40220, 2018.

[45] J. Zhang, J. Xu, W. Guan, and G. Du, "Damage detection of concrete-filled square steel tube (CFSST) column joints under cyclic loading using piezoceramic transducers," Sensors, vol. 18, no. 10, p. 3266, 2018

[46] Y. Moslehy, H. Gu, A. Belarbi, Y. L. Mo, and G. Song, "Smart aggregate based damage detection of circular RC columns under cyclic combined loading," Smart Materials and Structures, vol. 19, no. 6, Article ID 065021, 2010.

[47] G. Lu, Y. Li, and G. Song, "A delay-and-Boolean-ADD imaging algorithm for damage detection with a small number of piezoceramic transducers," Smart Materials and Structures, vol. 25, no. 9, Article ID 095030, 2016.

[48] J. Xu, C. Wang, H. Li, C. Zhang, J. Hao, and S. Fan, "Health monitoring of bolted spherical joint connection based on active sensing technique using piezoceramic transducers," Sensors, vol. 18, no. 6, p. 1727, 2018.

[49] K. Xu, C. Ren, Q. Deng, Q. Jin, and X. Chen, "Real-time monitoring of bond slip between GFRP bar and concrete structure using piezoceramic transducer-enabled active sensing," Sensors, vol. 18, no. 8, p. 2653, 2018.

[50] L. Huo, F. Wang, H. Li, and G. Song, "A fractal contact theory based model for bolted connection looseness monitoring using piezoceramic transducers," Smart Materials and Structures, vol. 26, no. 10, Article ID 104010, 2017.

[51] F. Wang, L. Huo, and G. Song, "A piezoelectric active sensing method for quantitative monitoring of bolt loosening using energy dissipation caused by tangential damping based on the fractal contact theory," Smart Materials and Structures, vol. 27, no. 1, Article ID 015023, 2017.

[52] L. Huo, D. Chen, Y. Liang, H. Li, X. Feng, and G. Song, "Impedance based bolt pre-load monitoring using piezoceramic smart washer," Smart Materials and Structures, vol. 26, no. 5, Article ID 057004, 2017.

[53] G. Lu, Y. Li, T. Wang, H. Xiao, L. Huo, and G. Song, "A multidelay-and-sum imaging algorithm for damage detection using piezoceramic transducers," Journal of Intelligent Material Systems and Structures, vol. 28, no. 9, pp. 1150-1159, 2017.

[54] B. Chen, C. Hei, M. Luo, M. S. C. Ho, and G. Song, "Pipeline two-dimensional impact location determination using time of arrival with instant phase (TOAIP) with piezoceramic transducer array," Smart Materials and Structures, vol. 27, no. 10, Article ID 105003, 2018.

[55] G. Zhang, J. Zhu, Y. Song, C. Peng, and G. Song, "A time reversal based pipeline leakage localization method with the adjustable resolution," IEEE Access, vol. 6, pp. 26993-27000, 2018. 